\title{
ISO LWS Spectra of T Tauri and Herbig AeBe stars ${ }^{\star}$
}

\author{
M. J. Creech-Eakman ${ }^{1,2}$, E. I. Chiang ${ }^{3}$, R. M. K. Joung ${ }^{4}$, G. A. Blake ${ }^{1}$, and E. F. van Dishoeck ${ }^{5}$ \\ 1 Division of Geological and Planetary Sciences, California Institute of Technology, MS 150-21, Pasadena, \\ CA 91125, USA \\ 2 Earth and Space Sciences Division, California Institute of Technology, Jet Propulsion Lab, MS 171-113, \\ Pasadena, CA 91109, USA \\ 3601 Campbell Hall, UC Berkeley Astronomy, Berkeley, CA 94720, USA \\ 4 Department of Astronomy, Columbia University, New York, NY 10027, USA \\ 5 Sterrewacht Leiden, PO Box 9513, 2300 RA, Leiden, The Netherlands
}

Received 29 August 2001 / Accepted 24 January 2002

\begin{abstract}
We present an analysis of ISO-LWS spectra of eight T Tauri and Herbig AeBe young stellar objects. Some of the objects are in the embedded phase of star-formation, whereas others have cleared their environs but are still surrounded by a circumstellar disk. Fine-structure lines of [OI] and [CII] are most likely excited by far-ultraviolet photons in the circumstellar environment rather than high-velocity outflows, based on comparisons of observed line strengths with predictions of photon-dominated and shock chemistry models. A subset of our stars and their ISO spectra are adequately explained by models constructed by Chiang \& Goldreich (1997) and Chiang et al. (2001) of isolated, passively heated, flared circumstellar disks. For these sources, the bulk of the LWS flux at wavelengths longward of $55 \mu \mathrm{m}$ arises from the disk interior which is heated diffusively by reprocessed radiation from the disk surface. At $45 \mu \mathrm{m}$, water ice emission bands appear in spectra of two of the coolest stars, and are thought to arise from icy grains irradiated by central starlight in optically thin disk surface layers.
\end{abstract}

Key words. stars: pre-main sequence - infrared: stars - line: identification - stars: formation

\section{Introduction}

One of the most rapidly developing areas in astrophysics is the study of the formation of stars and planetary systems. Through a combination of new high-sensitivity and highangular-resolution observational tools and detailed theoretical models, our understanding of young stellar objects (YSOs) and their attendant accretion disks has increased substantially (e.g. Adams \& Lin 1993; Beckwith \& Sargent 1996; Li \& Shu 1996; Mundy et al. 2000; McCaughrean et al. 2000). One of the major topics to be addressed concerns the physical and chemical evolution of the gas and dust in forming planetary systems (cf. Langer et al. 2000; van Dishoeck \& Blake 1998). What sets critical disk properties of size, accretion rate, variation of surface density with radius, radial and vertical temperature profiles, and gas vs. dust survival time scales? Questions such as these must be answered to provide the context for the formation of extra-solar planets (e.g. Marcy \& Butler

\footnotetext{
Send offprint requests to: M. J. Creech-Eakman, e-mail: mce@huey.jpl.nasa.gov

* Based in part on observations with ISO, an ESA project with instruments funded by ESA Member States (especially the PI countries: France, Germany, The Netherlands, and the United Kingdom) and with participation of ISAS and NASA.
}

1996). Spectroscopic observations of gas and dust at mid- through far-infrared wavelengths can help constrain these properties.

Data from the Infrared Space Observatory (ISO) Short Wavelength Spectrometer (SWS) and Long Wavelength Spectrometer (LWS) have enabled a new approach to characterizing circumstellar disks around YSOs that utilize moderate to high resolution SED spectra across the near- to far-infrared regions. When combined with archival data at optical through radio wavelengths, such complete spectra have not only better characterized the sizes and physical conditions in disks, but have also allowed the identification of solid state products such as crystalline silicates and water ice (Malfait et al. 1998; Waelkens et al. 1996, 1997; Nisini et al. 1996; Ceccarelli et al. 1997). Early and recent ISO results confirm the importance of complete spectral scans in studying YSOs. High resolution studies with HST and ground-based interferometric techniques have recently enabled several YSO/disk systems to be imaged directly (Burrows et al. 1996; Koresko 1998; Koerner \& Sargent 1995; Dutrey et al. 1996; Guilloteau et al. 1998), but extracting the physical and chemical conditions in the circumstellar material from such images can be difficult. 
Table 1. Stars in the sample.

\begin{tabular}{|c|c|c|c|c|c|c|c|c|c|c|c|}
\hline Star & Alt. Names & YSO Type & Sp. Type ${ }^{a}$ & $\begin{array}{c}F_{\text {IRAS }} \\
12 \mu \mathrm{m}\end{array}$ & $\begin{array}{r}(\mathrm{Jy}) \\
25 \mu \mathrm{m}\end{array}$ & $60 \mu \mathrm{m}$ & $100 \mu \mathrm{m}$ & $\begin{array}{l}F_{1.3 \mathrm{~mm}} \\
(\mathrm{mJy})\end{array}$ & $\begin{array}{c}\text { Rad. }^{b} \\
(\mathrm{AU})\end{array}$ & $\begin{array}{r}\text { Dist. }^{c} \\
(\mathrm{pc})\end{array}$ & $\begin{array}{r}\text { Age } \\
(\mathrm{Myr})\end{array}$ \\
\hline AA Tau & IRAS $04318+2422$ & $\mathrm{~T}$ Tauri & M0Ve & 0.28 & 0.42 & 0.93 & 9.60 & $88 \pm 9(2)$ & $150(3)$ & 140 & \\
\hline GG Tau & IRAS $04296+1725$ & $\mathrm{~T}$ Tauri & $\mathrm{K} 6 \mathrm{Ve}$ & 0.89 & 1.18 & 2.30 & 4.73 & $593 \pm 53(2)$ & $800(6)$ & 140 & \\
\hline AB Aur & MWC93, HD 31293 & Herbig & $\mathrm{B} 9 / \mathrm{A} 0 \mathrm{e}$ & 18.73 & 34.11 & 80.00 & 104.68 & $103 \pm 18(4)$ & $\leq 280$ & 149 & $2-5$ \\
\hline CQ Tau & HD 36910, BD+24 873 & Herbig & $\mathrm{A} 8 \mathrm{Ve} / \mathrm{F} 2 \mathrm{IVe}$ & 4.40 & 14.70 & 16.58 & 12.52 & $221 \pm 40(4)$ & 100 & $150(7)$ & $9(7)$ \\
\hline HD 36112 & MWC $758, \mathrm{BD}+25843$ & Herbig & A3e/A5IVe & 3.86 & 8.93 & 21.20 & 17.93 & $72 \pm 13(4)$ & $\leq 290$ & 205 & $3-6$ \\
\hline $\mathrm{LkH} \alpha 233$ & V375 Lac & Herbig & $\mathrm{A} 7 \mathrm{e}$ & $\cdots$ & . & $\cdots$ & $\cdots$ & $\cdots$ & $\cdots$ & $\cdots$ & $\cdots$ \\
\hline MWC 340 & V1685 Cyg, BD+40 4124 & Herbig & B2eq & 45.03 & 77.45 & 350.45 & 700.18 & 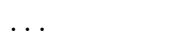 & $\ldots$ & 108 & $\ldots$ \\
\hline MWC 480 & HD 31648, BD+29 774 & Herbig & A2/3ep & 7.03 & 7.30 & 8.41 & 11.47 & $360 \pm 24(4)$ & $\leq 230$ & 131 & $3-6$ \\
\hline
\end{tabular}

${ }^{a}$ Spectral types from Thé et al. (1994) for Herbig AeBes and SIMBAD for T Tauris.

${ }^{b}$ Radii from mm dust measurements by Mannings \& Sargent (1997) unless noted.

${ }^{c}$ From Hipparcos parallax or Kenyon et al. (1994) unless noted.

(1) Mannings \& Sargent (1997); (2) van den Ancker et al. (2000); (3) Beckwith et al. (1990); (4) Dutrey et al. (1996); (5) Mannings \& Sargent (1997); (6) Dutrey et al. (1996); (7) Testi et al. (2001).

One question that can be answered directly through high signal-to-noise mid- and far-infrared spectra is the excitation mechanisms and gas temperatures present in YSOs. Through the determination of the line strengths of the fine-structure atomic lines of [CII] at $158 \mu \mathrm{m}$ and [OI] at 63 and $145 \mu \mathrm{m}$, and through a comparison of line ratios to the predictions of photon dominated region (PDR) models, it can be ascertained whether ultraviolet photons from the star (or star/disk boundary layer) or shocks constitute the dominant excitation mechanism. A similar study was performed for a group of Herbig AeBe stars by Lorenzetti et al. (1999) and Gianinni et al. (1999) and thus is motivated here for this sample, which on average contains less luminous objects. Likewise, investigations of rotational transistions of molecular lines of $\mathrm{CO}$ and $\mathrm{OH}$ allow one to probe the chemistry and physical parameters of circumstellar material, provided they are observed with high signal-to-noise and, ultimately, high spectral and spatial resolution.

Solid state materials, such as crystalline silicates and water ice, are not observed in the diffuse or dense interstellar medium, but are commonly observed in primitive solar system materials such as meteorites and comets. Detailed modelling of the SEDs, including the wavelengthdependent opacities of the likely constituents, will allow us to make quantitative estimates of the dust and ice masses present in YSOs. Combining these data with the observations of gas phase tracers will then permit the first complete census of the chemical environment of the circumstellar matter in the disks and envelopes around young stars.

Here we present an analysis of ISO-LWS data on a group of eight YSOs from a variety of spectral and Lada classes (Lada 1991), which indicate the degree of embeddedness of the YSO. In Sect. 2 of this paper we discuss our sample and the data reduction strategy. In Sect. 3 we present the spectral lines that have been positively identified and make conclusions about their excitation mechanisms based on comparisons to physical models. We demonstrate in Sect. 4 the utility of SED fits to the ISO-LWS data and the need for crystalline solid-state materials to reproduce various broad spectral features seen in many of these sources. In the final section we restate our conclusions based on this work.

\section{Observations and data}

\subsection{Sample}

The data sample consists of eight YSOs, two of which are T Tauri stars. The remainder are Herbig AeBe stars, which in this context refers to the fact that these systems are intermediate mass counterparts to TéTauri stars, and not necessarily all A or B spectral types. The T Tauri stars consist of AA Tau and GG Tau (a quadruple system), both Class II YSOs. Among the six Herbig AeBe stars are AB Aur, CQ Tau, HD 36112, MWC 480, MWC 340 and $\mathrm{LkH} \alpha$ 233. MWC $340(\mathrm{BD}+404124)$ and $\mathrm{LkH} \alpha$ 233 are embedded young stellar objects which are in the process of clearing their envelope material, whereas $\mathrm{AB}$ Aur has a tenuous residual envelope on a $10^{\prime \prime}$ scale (Grady et al. 1999). The long-wavelength emission of the remaining objects is dominated by circumstellar disks, largely free from surrounding cloud material. The ISOLWS spectrum of MWC 340 has previously been discussed by van den Ancker, Wesselius \& Tielens (2000), whereas that of AB Aur is also presented by Bouwman et al. (2000) and van den Ancker et al. (2000). Because our sample covers a wide range in spectral types (B through $\mathrm{M}$ ), YSO classes, and hence possible spectral energy distributions, a range of spectral signatures are to be expected for these stars. Table 1 contains a summary of the stars in the sample. 


\subsection{ISO LWS instrument}

We have observed a sample of eight YSOs using the Long Wavelength Spectrometer (LWS: Clegg et al. 1996) aboard the Infrared Space Observatory (ISO: Kessler et al. 1996) in the full grating scan mode (LWS AOT01). This configuration provides coverage from 43 to $197 \mu \mathrm{m}$ at a resolving power of $\simeq 200$, with an instrumental beam size of $\simeq 80^{\prime \prime}$. The spectra were processed using a modified version of the LIA portion of the data and the off-line pipeline version 7 (OLP V7 - see Sect. 2.3 below). The flux level and spectral responsivity calibrations are based on observations of Uranus, Mars and Neptune and comparisons of these observations with spectral models, resulting in an estimated flux accuracy of 30\% (Swinyard et al. 1996). The anticipated wavelength accuracy is $0.07 \mu \mathrm{m}$ in the short-wavelength range $(43-90 \mu \mathrm{m})$ and $0.15 \mu \mathrm{m}$ in the long-wavelength range (90-197 $\mu \mathrm{m})$.

Each spectrum is composed of subspectra from ten separate detectors. Flux responsivity of the detectors is ascertained by illuminator lamp flashes from calibration lamps located near the detectors on-board the LWS instrument. For the data presented here, spectral scans consisted of 7 to 14 grating scans (up and down) of the source flux across these ten detectors. Typical integration times were $\sim 2000$ s per spectrum. Because the sources in this sample were chosen to be as isolated as possible, off position measurements were not taken.

\subsection{Data reduction details}

Spectra were reduced using the LWS Interactive Analysis (LIA) package, version 7.2a, and the ISO Spectral Analysis Package (ISAP) version 1.6a. Spectra for each source were reduced in a similar manner to allow valid intercomparison of the results. A typical reduction consisted of six or seven steps using both LIA and ISAP. Because the ISO spectral reduction is sufficiently complicated and the methods to obtain the most reasonable spectra are still under investigation by a number of different groups, we describe next the steps used in a typical reduction of the LWS data.

Using LIA, approximations to the dark current DC levels for each detector in the spectra were derived and compared to the values obtained by the standard pipeline product using the IA_DARK routine. Occasionally strong drifts or glitches were found that were inadequately accounted for by the standard pipeline. These DC dark current levels were then recalculated based on estimates of what was judged to be the best data in a scan. Next, IA_DRIFT in LIA was used to identify glitch-free regions of each scan (called key points), from which a linear interpolation of the responsivity between up and down scans was accomplished. Finally, IA_ABSCORR was utilized to fit an absolute responsivity correction for the detectors by examining the illuminator lamp flash responses of each of the detectors during the calibration portions of the spectral scans. At each stage in the reduction, comparison to the standard pipeline product was made and adjustments were recorded. New LSAN files were created by these reductions and subsequently used in ISAP for the final data reduction.

In ISAP, a standard reduction consisted of three or four steps. Each detector spectrum was examined by hand in its up and down scans separately. All glitches and their subsequent decays were then clipped. A special median clip (where the highest and lowest values of the aggregate up and down scans are discarded and a $2.5 \sigma$ clipping is then applied to the remaining data) and an average to the mean of the subscans was next applied, using the anticipated spectral resolution bins as calculated by the ISAP tool. It was at this stage that significant spectral fringing in the long-wavelength detectors of bright sources was identified for fringe removal. Defringing was accomplished using the ISAP LWS defringe routine, which applied a Fourier Transform to the data to determine the period and phase of the fringe and deconvolve it from each sub-spectrum.

The ten individually averaged detectors were then plotted together and the relative flux differences between detectors were examined. These offsets arise from two main factors: (1) low flux sources for which dark currents are an appreciable amount of the actual flux levels, and (2) mispointings of the satellite, due to inaccurate coordinates in one case, which cause uneven illumination of the detectors. In order to produce reliable spectra, two or three relatively noise-free detectors near the center of the $45-200 \mu \mathrm{m}$ band were identified as fiducial subspectra. A gain correction to the dark current levels was calculated for the remaining subspectra using ISAP_SHIFT routine in order to rescale their overall flux levels to make overlapping spectral regions (usually $2-4 \mu \mathrm{m}$ of overlap) coincident and to merge all the subspectra together. In order to ascertain which merged version produced the best overall spectrum, sub-spectra were integrated over the IRAS 60 and $100 \mu \mathrm{m}$ filter functions. The subsequent photometry was compared to IRAS photometry where available, and the correctly merged spectrum was identified as the one which preserved the overall flux slope and level best when compared to IRAS photometry.

The merged subspectra were smoothed into $0.1 \mu \mathrm{m}$ bins using a weighted Gaussian algorithm (to preserve line shapes) and then resampled onto a $0.2 \mu \mathrm{m}$ grid. This final spectral product for each scan can be seen in order of flux strength in Fig. 1 (a-h).

One final note that ISO fluxes were found to be greater than the corresponding IRAS fluxes at 60 and $100 \mu \mathrm{m}$ by factors of $\sim 2-3$ in 3 out of the 4 sources (AA Tau, CQ Tau, and MWC 480) which we fitted with SED models (see below). Because it was not possible to fit both the slope and overall photometric level of the IRAS photometry for these sources, preference was given to preserving the character of the slope. Where there were additional discrepancies, preference was given to the ISO-LWS data for which the beam area is $\sim 2$ times smaller than that of IRAS. Note that for HD 36112 there is excellent agreement between ISO and IRAS. In fitting the spectra, 

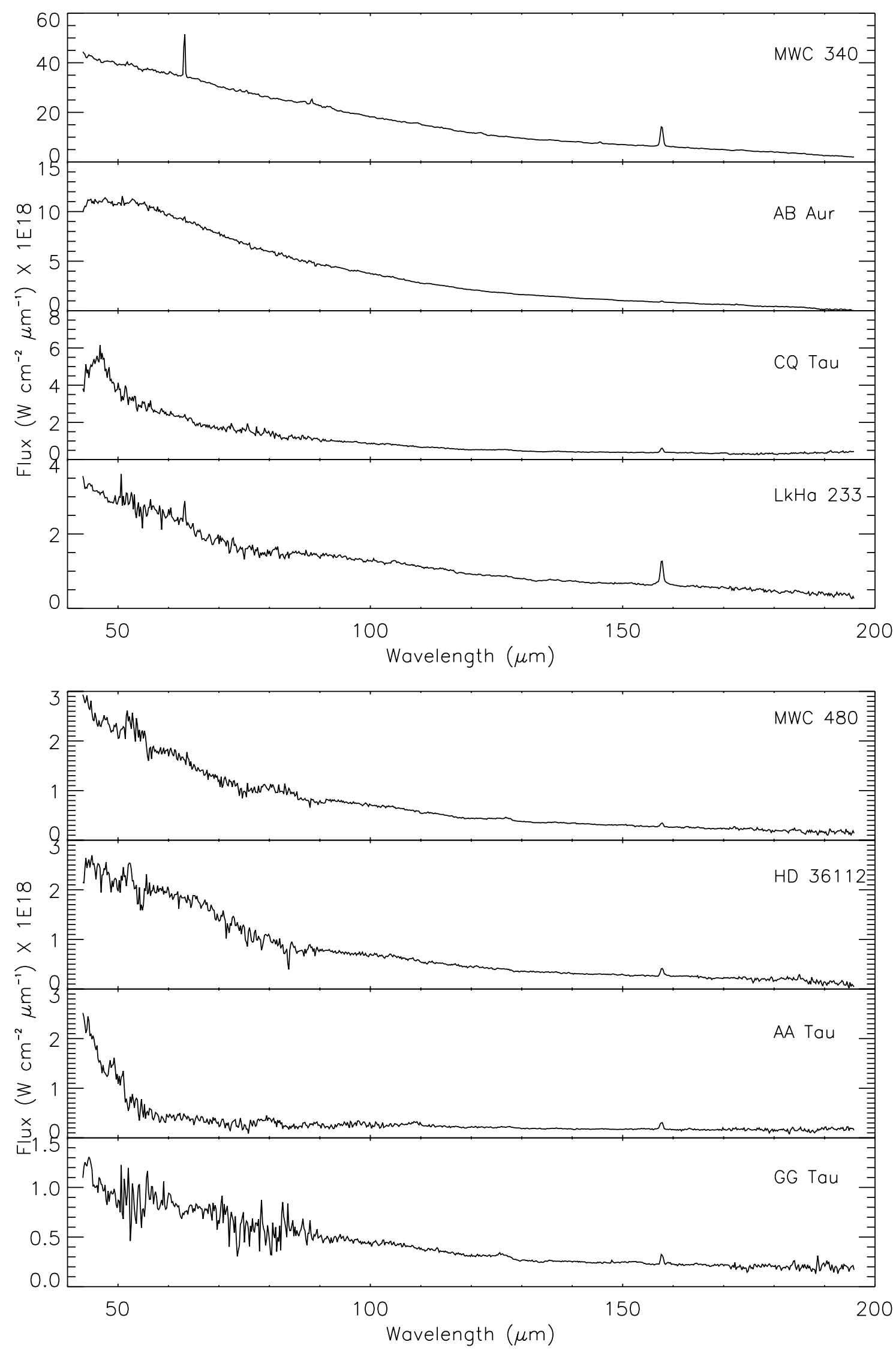

Fig. 1. Shown here are the eight ISO-LWS spectra for the YSOs in this sample. The subspectra have been merged to a fiducial spectrum (as described in the text) and smoothed onto a $0.1 \mu \mathrm{m}$ grid using a weighted Gaussian function to preserve line shapes. In most sources, the $158 \mu \mathrm{m}$ [CII] line is evident, along with as the $63 \mu \mathrm{m}$ [OI] line, particularly in MWC 340. 
preference was also given to the central portions of the ISO scans between 50 and $170 \mu \mathrm{m}$ where individual detectors overlap in wavelength coverage, for which measured fluxes are consequently deemed more reliable.

\subsection{Background contributions}

In order to ascertain whether or not there is significant background contamination in this ISO-LWS dataset, we pursued three different avenues of investigation to determine the background contributions to the data presented herein. First, COBE pointings were retrieved for each source from the COBE DIRBE database maintained at GSFC for 60, 100, 140, and $240 \mu \mathrm{m}$ filters encompassing days $0-240$ of the COBE mission (http://space.gsfc.nasa.gov/astro/cobe/ dirbe_products.html). The backgrounds for an $80^{\prime \prime}$ LWS beam were calculated from these backgrounds for each source. For all the stars except BD +40 4124 the background calculated using this method was less than $3 \mathrm{Jy}$ for $\lambda \leq 100 \mu \mathrm{m}\left(0.9 \mathrm{E}-19 \mathrm{~W} \mathrm{~cm}^{-2} \mu \mathrm{m}^{-1}\right.$ at $\left.100 \mu \mathrm{m}\right)$ and 6 Jy for $100 \leq \lambda \leq 240 \mu \mathrm{m}\left(0.45 \mathrm{E}-19 \mathrm{~W} \mathrm{~cm}^{-2} \mu \mathrm{m}^{-1}\right.$ at $200 \mu \mathrm{m})$. For $\mathrm{BD}+404124$ these values were 40 and $60 \mathrm{Jy}$ respectively. Our second level of investigation involved inspection of the ISO-PHOT data using the ESA inspection tools online for data which has not been scientifically validated (http://www.iso.vilspa.esa.es). Unfortunately, only three stars in this paper were also imaged with ISO PHOT, AA Tau, AB Aur and LkH $\alpha 233$. The extrapolated values between the COBE DIRBE filter backgrounds and the backgrounds of the fields for the ISO-PHOT data agree within $1 \mathrm{Jy}$ of each other. Finally, we used the IRSA tool at Caltech to examine the 0.5 degree square fields around each star from the IRAS database at 12, 25, 60 and $100 \mu \mathrm{m}$ (http://irsa.ipac.caltech.edu). These data showed that most of the fields contain no other identified point source at any of the IRAS wavelengths which is within $10^{\prime}$ of the YSO, with the exception of two sources. For AB Aur, there is one close infrared source in the field at about 4 arcmin distant (too far to contribute to the ISO-LWS beam). Upon further investigation, this source can positively be identified to be SU Aur. For $\mathrm{BD}+404124$, there are 16 other IRAS point sources in the 0.5 square degree beam examined with IRSA. None of these sources appears to be closer than $40^{\prime \prime}$ to the YSO which would render them outside of the ISO-LWS beam; however it is clear that with this plethora of sources in the $\mathrm{BD}+404124$ field that the backgrounds in the COBE DIRBE beam would be significantly contaminated, which accounts for the much higher backgrounds seen by COBE DIRBE for this source compared to the other seven. Based on these investigations and private communications from other ISO investigators (see below), we have not made any global adjustments to the background levels for these ISO-LWS data.

\section{Spectral lines}

Through the identification of forbidden fine structure lines (principally of [OI] and [CII]) and from weak detections or upper bounds to molecular rotational transitions (principally of $\mathrm{CO}, \mathrm{OH}$, and $\mathrm{H}_{2} \mathrm{O}$ ), limits can be placed on the various excitation mechanisms present in low- to moderate-mass YSOs. Large gradients in the physical conditions exist in such objects, and a number of energetic sources such as ultraviolet photolysis, shocks, and high temperatures in the regions closest to the embedded objects, can both excite a wide range of transitions and substantially alter the composition of the material from which the star is assembled. The ISO-LWS spectra contain features from both gas phase spectral lines and the spectrally broader resonances of solid state materials and ices in the dust. We outline first the spectral line results before turning to a discussion of the SED fits and the role of solid state features therein.

\subsection{Line identification}

Line identification involved three steps. First, the individual detector spectra were examined by hand after fitting and subtracting a second or third order polynomial to the continuum level for the entire detector sub-spectrum. Simple gaussian fits were then attempted for any features which resembled a spectral profile. A list was generated which included lines having a signal-to-noise $\geq 2.0$ and a FWHM comparable to the instrumental resolution element (i.e. $0.29 \mu \mathrm{m} \pm 0.15 \mu \mathrm{m}$ for $\lambda \leq 90 \mu \mathrm{m}$ and $0.60 \mu \mathrm{m} \pm$ $0.20 \mu \mathrm{m}$ for $\lambda \geq 90 \mu \mathrm{m})$. Lines found to be somewhat larger than the instrumental $F W H M$ are likely accounted for by $\Lambda$ doubling splitting of the levels (e.g. for $\mathrm{OH}$ in particular) as was seen by Giannini et al. (1999) or by blends of closely-spaced transitions from different species. If lines were found to be only marginally larger or smaller than the instrumental resolution but fitting all other criteria, these lines were reported in the line tables (Tables 2-3) and their anomalous widths notated.

These lines were identified using the spectral line lists endemic to the ISAP routine. For those lines unidentified in the ISAP spectral line reduction tools, an IDL program was written which matched up all lines among the eight sources which were within $0.1 \mu \mathrm{m}$ bins for $\lambda \leq 90 \mu \mathrm{m}$ and $0.2 \mu \mathrm{m}$ bins for $\lambda \geq 90 \mu \mathrm{m}$. The program further reported the average line center and number of sources among the eight for which a line was found that matched these criteria. In total, there was no coincidence of the same spectral line found in three or more sources, which met the above criteria and were unidentified in the ISAP spectral analysis line list. Therefore, we choose not to attempt to identify any line features not identified by the ISAP spectral line tool. Further, we note that the emission from species such as $\mathrm{CO}, \mathrm{OH}$, and $\mathrm{H}_{2} \mathrm{O}$ is often at or below the sensitivity threshold of the ISO-LWS measurements. When a plethora of lines of a particular species are identified for an individual source, it increases the likelihood that the 
Table 2. Atomic and fine structure lines.

\begin{tabular}{|c|c|c|c|c|c|}
\hline Star & $\begin{array}{r}\text { Line Center } \\
(\mu \mathrm{m}) \\
\end{array}$ & $\begin{array}{r}\text { (Base) Line Strength } \\
\left(\mathrm{W} \mathrm{cm} \mathrm{cm}^{-2} \times 10^{-19}\right) \\
\end{array}$ & Identified Species & Transition & Notes \\
\hline \multicolumn{6}{|c|}{ AA Tau } \\
\hline & 63.18 & $\leq 1.50$ & {$[\mathrm{OI}]$} & $\left({ }^{3} \mathrm{P}_{1}-{ }^{3} \mathrm{P}_{2}\right)$ & $\mathrm{e}$ \\
\hline & $76.27 \pm 0.01$ & $0.61 \pm 0.08$ & $\mathrm{HI}$ & $(23-18)$ & \\
\hline & 145.52 & $\leq 0.42$ & {$[\mathrm{OI}]$} & $\left({ }^{3} \mathrm{P}_{0}-{ }^{3} \mathrm{P}_{1}\right)$ & $\mathrm{e}$ \\
\hline & $157.69 \pm 0.02$ & $1.13 \pm 0.10$ & [CII] & $\left({ }^{2} \mathrm{P}_{3 / 2}{ }^{-2} \mathrm{P}_{1 / 2}\right)$ & \\
\hline \multicolumn{6}{|c|}{ GG Tau } \\
\hline & 63.18 & $\leq 1.29$ & {$[\mathrm{OI}]$} & $\left({ }^{3} \mathrm{P}_{1}-{ }^{3} \mathrm{P}_{2}\right)$ & e \\
\hline & 145.52 & $\leq 0.08$ & {$[\mathrm{OI}]$} & $\left({ }^{3} \mathrm{P}_{0}-{ }^{3} \mathrm{P}_{1}\right)$ & $\mathrm{e}$ \\
\hline & $157.77 \pm 0.03$ & $0.59 \pm 0.09$ & {$[\mathrm{CII}]$} & $\left({ }^{2} \mathrm{P}_{3 / 2}{ }^{2} \mathrm{P}_{1 / 2}\right)$ & \\
\hline \multicolumn{6}{|c|}{ AB Aur } \\
\hline & $63.18 \pm 0.02$ & $1.04 \pm 0.26$ & {$[\mathrm{OI}]$} & $\left({ }^{3} \mathrm{P}_{1}-{ }^{3} \mathrm{P}_{2}\right)$ & \\
\hline & 145.52 & $\leq 1.32$ & {$[\mathrm{OI}]$} & $\left({ }^{3} \mathrm{P}_{0}{ }^{-3} \mathrm{P}_{1}\right)$ & $\mathrm{e}$ \\
\hline & $157.77 \pm 0.02$ & $0.71 \pm 0.07$ & [CII] & $\left({ }^{2} \mathrm{P}_{3 / 2}{ }^{-2} \mathrm{P}_{1 / 2}\right)$ & \\
\hline \multicolumn{6}{|c|}{ CQ Tau } \\
\hline & $60.41 \pm 0.02$ & $0.43 \pm 0.15$ & HeII & $(23-21)$ & \\
\hline & $63.18 \pm 0.01$ & $0.51 \pm 0.10$ & {$[\mathrm{OI}]$} & $\left({ }^{3} \mathrm{P}_{1}-{ }^{3} \mathrm{P}_{2}\right)$ & \\
\hline & 145.52 & $\leq 0.23$ & {$[\mathrm{OI}]$} & $\left({ }^{3} \mathrm{P}_{0^{-}}{ }^{3} \mathrm{P}_{1}\right)$ & $\mathrm{e}$ \\
\hline & $157.77 \pm 0.01$ & $1.73 \pm 0.07$ & {$[\mathrm{CII}]$} & $\left({ }^{2} \mathrm{P}_{3 / 2}{ }^{-2} \mathrm{P}_{1 / 2}\right)$ & \\
\hline \multicolumn{6}{|c|}{ HD 36112} \\
\hline & 63.18 & $\leq 2.16$ & {$[\mathrm{OI}]$} & $\left({ }^{3} \mathrm{P}_{1}{ }^{-3} \mathrm{P}_{2}\right)$ & $\mathrm{e}$ \\
\hline & $111.20 \pm 0.03$ & $0.21 \pm 0.06$ & $\mathrm{HI}$ & $(18-16)$ & $\mathrm{a}$ \\
\hline & 145.52 & $\leq 0.31$ & {$[\mathrm{OI}]$} & $\left({ }^{3} \mathrm{P}_{0}{ }^{-3} \mathrm{P}_{1}\right)$ & $\mathrm{e}$ \\
\hline & $157.80 \pm 0.02$ & $1.08 \pm 0.10$ & [CII] & $\left({ }^{2} \mathrm{P}_{3 / 2}{ }^{-2} \mathrm{P}_{1 / 2}\right)$ & \\
\hline \multicolumn{6}{|c|}{$\mathrm{LkH} \alpha 233$} \\
\hline & $60.42 \pm 0.02$ & $0.70 \pm 0.25$ & HeII & $(23-21)$ & \\
\hline & $63.20 \pm 0.01$ & $2.11 \pm 0.21$ & {$[\mathrm{OI}]$} & $\left({ }^{3} \mathrm{P}_{1}{ }^{-3} \mathrm{P}_{2}\right)$ & \\
\hline & $95.03 \pm 0.10$ & $0.36 \pm 0.09$ & HI & $(20-17)$ & d \\
\hline & $145.32 \pm 0.04$ & $0.44 \pm 0.58$ & {$[\mathrm{OI}]$} & $\left({ }^{3} \mathrm{P}_{0}-{ }^{3} \mathrm{P}_{1}\right)$ & $\mathrm{c}$ \\
\hline & $157.75 \pm 0.02$ & $4.63 \pm 0.20$ & {$[\mathrm{CII}]$} & $\left({ }^{2} \mathrm{P}_{3 / 2}-{ }^{2} \mathrm{P}_{1 / 2}\right)$ & \\
\hline \multicolumn{6}{|c|}{ MWC 340(f) } \\
\hline & $51.81 \pm 0.02$ & $7.38 \pm 1.50$ & [OIII $]$ & $\left({ }^{3} \mathrm{P}_{2}{ }^{-3} \mathrm{P}_{1}\right)$ & \\
\hline & $63.17 \pm 0.01$ & $67.35 \pm 3.51$ & {$[\mathrm{OI}]$} & $\left({ }^{3} \mathrm{P}_{1}{ }^{-3} \mathrm{P}_{2}\right)$ & \\
\hline & $78.54 \pm 0.03$ & $4.98 \pm 1.07$ & HeII & $(25-23)$ & $\mathrm{b}$ \\
\hline & $88.38 \pm 0.01$ & $8.50 \pm 0.84$ & [OIII $]$ & $\left({ }^{3} \mathrm{P}_{1}-{ }^{3} \mathrm{P}_{0}\right)$ & \\
\hline & $121.86 \pm 0.04$ & $4.56 \pm 0.67$ & {$[\mathrm{NII}]$} & $\left({ }^{3} \mathrm{P}_{1}-{ }^{3} \mathrm{P}_{2}\right)$ & \\
\hline & $145.51 \pm 0.02$ & $2.77 \pm 0.26$ & {$[\mathrm{OI}]$} & $\left({ }^{3} \mathrm{P}_{0}-{ }^{3} \mathrm{P}_{1}\right)$ & \\
\hline & $157.74 \pm 0.01$ & $57.00 \pm 0.86$ & {$[\mathrm{CII}]$} & $\left({ }^{2} \mathrm{P}_{3 / 2}{ }^{2} \mathrm{P}_{1 / 2}\right)$ & \\
\hline & $167.50 \pm 0.03$ & $1.19 \pm 0.21$ & HeII & $(25-24)$ & \\
\hline \multicolumn{6}{|c|}{ MWC 480} \\
\hline & $49.64 \pm 0.06$ & $0.39 \pm 0.12$ & $\mathrm{HI}$ & $(22-16)$ & d \\
\hline & 63.18 & $\leq 1.10$ & {$[\mathrm{OI}]$} & $\left({ }^{3} \mathrm{P}_{1}{ }^{-3} \mathrm{P}_{2}\right)$ & e \\
\hline & 145.52 & $\leq 0.18$ & {$[\mathrm{OI}]$} & $\left({ }^{3} \mathrm{P}_{0}-{ }^{3} \mathrm{P}_{1}\right)$ & e \\
\hline & $157.78 \pm 0.02$ & $0.59 \pm 0.06$ & {$[\mathrm{CII}]$} & $\left({ }^{2} \mathrm{P}_{3 / 2}{ }^{-2} \mathrm{P}_{1 / 2}\right)$ & \\
\hline
\end{tabular}

a - Line FWHM marginally smaller than instrumental.

b - Line FWHM marginally larger than instrumental.

c - Distance between rest and observed wavelength for line marginally exceeds resolution element.

d - Measured signal-to-noise for line between 2.0 and 3.0.

e - No line identified at this position. $3.0 \sigma \mathrm{rms}$ baseline flux reported in $\mathrm{W} \mathrm{cm}^{-2} \mu \mathrm{m}^{-1} \times 10^{-19}$.

f - Comparison of the line strengths identified here which were in common with van den Ancker et al. (2000) show general agreement in strengths at $10 \%$ level or below. Note also that van den Ancker et al. attribute all the [CII] flux to background.

transitions we are detecting are veritable. The coincidence of transitions of molecular species is discussed below in the subsection on molecular transitions.

\subsection{Fine-structure lines}

As was found by Lorenzetti et al. (1999) in their ISO-LWS spectra of Herbig AeBe stars, the fine-structure lines of 
Table 3. Molecular lines.

\begin{tabular}{|c|c|c|c|c|c|}
\hline Star & $\begin{array}{r}\text { Line Center } \\
(\mu \mathrm{m})\end{array}$ & $\begin{array}{l}\text { (Base) Line Strength } \\
\quad\left(\mathrm{W} \mathrm{cm}-2 \times 10^{-19}\right)\end{array}$ & Identified Species & Transition & Notes \\
\hline \multicolumn{6}{|c|}{ AA Tau } \\
\hline & 108.07 & $\leq 0.45$ & $\mathrm{o}-\mathrm{H}_{2} \mathrm{O}$ & $\left(2_{21}-1_{10}\right)$ & $\mathrm{b}$ \\
\hline & 119.33 & $\leq 0.36$ & $\mathrm{OH}_{3 / 2-3 / 2}$ & $\left(5 / 2^{+}, 2-3 / 2^{-}, 1\right)$ & $\mathrm{b}$ \\
\hline & & & $\mathrm{OH}_{3 / 2-3 / 2}$ & $\left(5 / 2^{+}, 3-3 / 2^{-}, 2\right)$ & $\mathrm{b}$ \\
\hline & 153.27 & $\leq 0.27$ & $\mathrm{CO}$ & $(17-16)$ & $\mathrm{b}$ \\
\hline & 174.63 & $\leq 0.60$ & $\mathrm{o}-\mathrm{H}_{2} \mathrm{O}$ & $\left(3_{03}-2_{12}\right)$ & $\mathrm{b}$ \\
\hline & 179.53 & $\leq 0.60$ & $\mathrm{o}-\mathrm{H}_{2} \mathrm{O}$ & $\left(2_{12}-1_{01}\right)$ & $\mathrm{b}$ \\
\hline & 186.00 & $\leq 1.10$ & $\mathrm{CO}$ & $(14-13)$ & $\mathrm{b}$ \\
\hline \multicolumn{6}{|c|}{ GG Tau } \\
\hline & 108.07 & $\leq 0.31$ & $\mathrm{o}-\mathrm{H}_{2} \mathrm{O}$ & $\left(2_{21}-1_{10}\right)$ & $\mathrm{b}$ \\
\hline & 119.33 & $\leq 0.29$ & $\mathrm{OH}_{3 / 2-3 / 2}$ & $\left(5 / 2^{+}, 2-3 / 2^{-}, 1\right)$ & $\mathrm{b}$ \\
\hline & & & $\mathrm{OH}_{3 / 2-3 / 2}$ & $\left(5 / 2^{+}, 3-3 / 2^{-}, 2\right)$ & $\mathrm{b}$ \\
\hline & 153.27 & $\leq 0.37$ & $\mathrm{CO}$ & $(17-16)$ & $\mathrm{b}$ \\
\hline & 174.63 & $\leq 0.68$ & $\mathrm{o}-\mathrm{H}_{2} \mathrm{O}$ & $\left(3_{03}-2_{12}\right)$ & $\mathrm{b}$ \\
\hline & 179.53 & $\leq 0.69$ & $\mathrm{o}-\mathrm{H}_{2} \mathrm{O}$ & $\left(2_{12}-1_{01}\right)$ & $\mathrm{b}$ \\
\hline & 186.00 & $\leq 0.68$ & $\mathrm{CO}$ & $(14-13)$ & $\mathrm{b}$ \\
\hline \multicolumn{6}{|c|}{ AB Aur } \\
\hline & 108.07 & $\leq 1.93$ & $\mathrm{o}-\mathrm{H}_{2} \mathrm{O}$ & $\left(2_{21}-1_{10}\right)$ & $\mathrm{b}$ \\
\hline & 119.33 & $\leq 0.45$ & $\mathrm{OH}_{3 / 2-3 / 2}$ & $\left(5 / 2^{+}, 2-3 / 2^{-}, 1\right)$ & $\mathrm{b}$ \\
\hline & & & $\mathrm{OH}_{3 / 2-3 / 2}$ & $\left(5 / 2^{+}, 3-3 / 2^{-}, 2\right)$ & $\mathrm{b}$ \\
\hline & 153.27 & $\leq 0.39$ & $\mathrm{CO}$ & $(17-16)$ & $\mathrm{b}$ \\
\hline & 174.63 & $\leq 1.06$ & $\mathrm{o}-\mathrm{H}_{2} \mathrm{O}$ & $\left(3_{03}-2_{12}\right)$ & $\mathrm{b}$ \\
\hline & 179.53 & $\leq 1.05$ & $\mathrm{o}-\mathrm{H}_{2} \mathrm{O}$ & $\left(2_{12}-1_{01}\right)$ & $\mathrm{b}$ \\
\hline & 186.00 & $\leq 1.06$ & $\mathrm{CO}$ & $(14-13)$ & $\mathrm{b}$ \\
\hline \multicolumn{6}{|c|}{ CQ Tau } \\
\hline & 108.07 & $\leq 0.63$ & $\mathrm{o}-\mathrm{H}_{2} \mathrm{O}$ & $\left(2_{21}-1_{10}\right)$ & $\mathrm{b}$ \\
\hline & 119.33 & $\leq 0.57$ & $\mathrm{OH}_{3 / 2-3 / 2}$ & $\left(5 / 2^{+}, 2-3 / 2^{-}, 1\right)$ & $\mathrm{b}$ \\
\hline & & & $\mathrm{OH}_{3 / 2-3 / 2}$ & $\left(5 / 2^{+}, 3-3 / 2^{-}, 2\right)$ & $\mathrm{b}$ \\
\hline & 153.27 & $\leq 0.15$ & $\mathrm{CO}$ & $(17-16)$ & $\mathrm{b}$ \\
\hline & 174.63 & $\leq 0.50$ & $\mathrm{o}-\mathrm{H}_{2} \mathrm{O}$ & $\left(3_{03}-2_{12}\right)$ & $\mathrm{b}$ \\
\hline & 179.53 & $\leq 0.50$ & $\mathrm{o}-\mathrm{H}_{2} \mathrm{O}$ & $\left(2_{12}-1_{01}\right)$ & $\mathrm{b}$ \\
\hline & 186.00 & $\leq 1.80$ & $\mathrm{CO}$ & $(14-13)$ & $\mathrm{b}$ \\
\hline \multicolumn{6}{|c|}{ HD 36112} \\
\hline & $108.28 \pm 0.08$ & $0.19 \pm 0.09$ & $\mathrm{o}-\mathrm{H}_{2} \mathrm{O}$ & $\left(2_{21}-1_{10}\right)$ & $\mathrm{a}$ \\
\hline & 119.33 & $\leq 0.36$ & $\mathrm{OH}_{3 / 2-3 / 2}$ & $\left(5 / 2^{+}, 2-3 / 2^{-}, 1\right)$ & $\mathrm{b}$ \\
\hline & & & $\mathrm{OH}_{3 / 2-3 / 2}$ & $\left(5 / 2^{+}, 3-3 / 2^{-}, 2\right)$ & $\mathrm{b}$ \\
\hline & 153.27 & $\leq 0.27$ & $\mathrm{CO}$ & $(17-16)$ & $\mathrm{b}$ \\
\hline & 174.63 & $\leq 0.60$ & $\mathrm{o}-\mathrm{H}_{2} \mathrm{O}$ & $\left(3_{03}-2_{12}\right)$ & $\mathrm{b}$ \\
\hline & 179.53 & $\leq 0.60$ & $\mathrm{o}-\mathrm{H}_{2} \mathrm{O}$ & $\left(2_{12}-1_{01}\right)$ & $\mathrm{b}$ \\
\hline & 186.00 & $\leq 1.10$ & $\mathrm{CO}$ & $(14-13)$ & $\mathrm{b}$ \\
\hline \multicolumn{6}{|c|}{$\mathrm{LkH} \alpha 233$} \\
\hline & 108.07 & $\leq 0.50$ & $\mathrm{o}-\mathrm{H}_{2} \mathrm{O}$ & $\left(2_{21}-1_{10}\right)$ & $\mathrm{b}$ \\
\hline & 119.33 & $\leq 0.30$ & $\mathrm{OH}_{3 / 2-3 / 2}$ & $\left(5 / 2^{+}, 2-3 / 2^{-}, 1\right)$ & $\mathrm{b}$ \\
\hline & & & $\mathrm{OH}_{3 / 2-3 / 2}$ & $\left(5 / 2^{+}, 3-3 / 2^{-}, 2\right)$ & $\mathrm{b}$ \\
\hline & 153.27 & $\leq 1.53$ & $\mathrm{CO}$ & $(17-16)$ & $\mathrm{b}$ \\
\hline & 174.63 & $\leq 1.05$ & $\mathrm{o}-\mathrm{H}_{2} \mathrm{O}$ & $\left(3_{03}-2_{12}\right)$ & $\mathrm{b}$ \\
\hline & 179.53 & $\leq 0.63$ & $\mathrm{o}-\mathrm{H}_{2} \mathrm{O}$ & $\left(2_{12}-1_{01}\right)$ & $\mathrm{b}$ \\
\hline & 186.00 & $\leq 0.68$ & $\mathrm{CO}$ & $(14-13)$ & $\mathrm{b}$ \\
\hline
\end{tabular}

[CII] $158 \mu \mathrm{m}$ and [OI] $63 \mu \mathrm{m}$ are generally the strongest and most evident lines in the YSOs spectra acquired here. Their relative line strengths can be used to determine the excitation mechanism for these species. Our sources were chosen to be isolated to the extent possible given the $80^{\prime \prime}$ ISO-LWS beam, and so off-pointings to determine background fluxes for [CII] were not obtained. Further investigation for evidence of background contamination was done using COBE DIRBE, ISO-PHOT data and IRAS data through the IRSA database tool, and we believe we can confidently claim that the YSOs presented in this paper are not significantly contaminated from background 
Table 3. continued.

\begin{tabular}{|c|c|c|c|c|c|}
\hline Star & $\begin{array}{r}\text { Line Center } \\
(\mu \mathrm{m})\end{array}$ & $\begin{array}{r}\text { (Base) Line Strength } \\
\left(\mathrm{W} \mathrm{cm}-2 \times 10^{-19}\right)\end{array}$ & Identified Species & Transition & Notes \\
\hline \multicolumn{6}{|c|}{ MWC 340(c) } \\
\hline & $77.092 \pm 0.028$ & $1.553 \pm 0.551$ & $\mathrm{CO}$ & $(34-33)$ & \multirow{3}{*}{$\mathrm{a}$} \\
\hline & $90.124 \pm 0.060$ & $1.122 \pm 0.549$ & $\mathrm{p}-\mathrm{H}_{2} \mathrm{O}$ & $\left(3_{22}-2_{11}\right)$ & \\
\hline & & & $\mathrm{CO}$ & $(29-28)$ & \\
\hline & $104.488 \pm 0.045$ & $2.057 \pm 0.637$ & $\mathrm{CO}$ & $(25-24)$ & \multirow{3}{*}{$\mathrm{b}$} \\
\hline & 108.07 & $\leq 2.70$ & $\mathrm{o}-\mathrm{H}_{2} \mathrm{O}$ & $\left(2_{21}-1_{10}\right)$ & \\
\hline & $119.437 \pm 0.127$ & $3.665 \pm 1.053$ & $\mathrm{OH}_{3 / 2-3 / 2}$ & $\left(5 / 2^{+}, 2-3 / 2^{-}, 1\right)$ & \\
\hline & & & $\mathrm{OH}_{3 / 2-3 / 2}$ & $\left(5 / 2^{+}, 3-3 / 2^{-}, 2\right)$ & \multirow{3}{*}{$\mathrm{a}$} \\
\hline & $123.958 \pm 0.064$ & $1.014 \pm 0.525$ & $\mathrm{CO}$ & $(21-20)$ & \\
\hline & $137.026 \pm 0.043$ & $1.858 \pm 0.353$ & $\mathrm{C}^{18} \mathrm{O}$ & $(20-19)$ & \\
\hline & & & $\mathrm{CO}$ & $(19-18)$ & \multirow[b]{2}{*}{$\mathrm{b}$} \\
\hline & 153.27 & $\leq 1.10$ & $\mathrm{CO}$ & $(17-16)$ & \\
\hline & $162.883 \pm 0.028$ & $1.270 \pm 0.273$ & $\mathrm{CO}$ & $(16-15)$ & \\
\hline & $173.611 \pm 0.039$ & $2.565 \pm 0.312$ & $\mathrm{CO}$ & $(15-14)$ & \\
\hline & & & $\mathrm{o}-\mathrm{H}_{2} \mathrm{O}$ & $\left(3_{03}-2_{12}\right)$ & \\
\hline & $179.413 \pm 0.046$ & $1.317 \pm 0.338$ & $\mathrm{o}-\mathrm{H}_{2} \mathrm{O}$ & $\left(2_{12}-1_{01}\right)$ & \\
\hline & $185.926 \pm 0.044$ & $0.952 \pm 0.341$ & $\mathrm{CO}$ & $(14-13)$ & $\mathrm{a}$ \\
\hline \multicolumn{6}{|c|}{ MWC 480} \\
\hline & 108.07 & $\leq 0.47$ & $\mathrm{o}-\mathrm{H}_{2} \mathrm{O}$ & $\left(2_{21}-1_{10}\right)$ & $\mathrm{b}$ \\
\hline & \multirow[t]{2}{*}{119.33} & \multirow[t]{2}{*}{$\leq 0.18$} & $\mathrm{OH}_{3 / 2-3 / 2}$ & $\left(5 / 2^{+}, 2-3 / 2^{-}, 1\right)$ & $\mathrm{b}$ \\
\hline & & & $\mathrm{OH}_{3 / 2-3 / 2}$ & $\left(5 / 2^{+}, 3-3 / 2^{-}, 2\right)$ & $\mathrm{b}$ \\
\hline & 153.27 & $\leq 0.31$ & $\mathrm{CO}$ & $(17-16)$ & $\mathrm{b}$ \\
\hline & 174.63 & $\leq 0.73$ & $\mathrm{o}-\mathrm{H}_{2} \mathrm{O}$ & $\left(3_{03}-2_{12}\right)$ & $\mathrm{b}$ \\
\hline & 179.53 & $\leq 0.73$ & $\mathrm{o}-\mathrm{H}_{2} \mathrm{O}$ & $\left(2_{12}-1_{01}\right)$ & $\mathrm{b}$ \\
\hline & 186.00 & $\leq 0.73$ & $\mathrm{CO}$ & $(14-13)$ & $\mathrm{b}$ \\
\hline
\end{tabular}

a - Signal-to-noise for line between 2.0 and 3.0.

$\mathrm{b}-$ No line identified at this position. $3.0 \sigma \mathrm{rms}$ baseline flux reported in $\mathrm{W} \mathrm{cm}^{-2} \mu \mathrm{m}^{-1} \times 10^{-19}$.

c - Comparison of the line strengths identified here which were in common with van den Ancker et al. (2000) (CO lines) show general agreement in strengths at $30 \%$ level.

emission (see Sect. 2.4). However, for the purposes of modelling we estimated the background contribution to the [CII] line strengths based on galactic models from COBE data using the following equation for the LWS beam (Bennett et al. 1994):

$F=1.7 \times 10^{-20} \csc |b|$

where $F$ is in $W \mathrm{~cm}^{-2}$ and $b$ is galactic latitude in degrees for sources where $|b| \geq 15$ degrees. For sources within 15 degrees of the galactic plane, the ISO-LWS measurement at $158 \mu \mathrm{m}$ served as an upper limit on the emission from the [CII] line. We believe this method of estimating the background is justified because Bock et al. (1993) find a warm ionized medium contribution to be nearly a factor of 10 lower than the upper limit of [CII] emission found by Bennett et al. from the COBE data. These measurements taken together suggest that the cold neutral medium dominates over the warm ionized medium in background contributions to the $[\mathrm{CII}]$ line emission. Of course, such a subtraction scheme will not account for residual extended cloud material that is no longer associated with the YSO but contained within the LWS beam. Typical [C II] fluxes measured with the LWS for cold cirrus material range are
$(2-3) \times 10^{-20} \mathrm{~W} \mathrm{~cm}^{-2}$ (R. Stark, private communication), whereas off positions in Taurus give $(5-6) \times 10^{-20} \mathrm{~W} \mathrm{~cm}^{-2}$ (D. Lorenzetti, private communication). Both values are below the measured fluxes for most of our sources.

In those cases where one or more of the above mentioned fine-structure lines were absent, the $3 \sigma \mathrm{rms}$ of the flux level at the position of line center was used as an upper limit to determine the excitation mechanism. Table 4 summarizes the [CII] estimated line strengths used to constrain the excitation mechanisms for stars with $|b|>15$ degrees, while the spectra of the $[\mathrm{CII}]$ and $[\mathrm{OI}]$ lines are presented in Fig. 2. These line strengths are used below to examine the potential roles of ultraviolet versus shock excitation of these intense far-infrared emitters.

\subsection{Modelling of Far-infrared Fine Structure Transitions}

In Fig. 3 the observed line intensity ratios for four sources are superimposed on a grid of PDR models by Kaufman et al. (1999). Differences between the line intensity 
Table 4. On-source estimated line strengths.

\begin{tabular}{|c|c|c|c|c|c|}
\hline Star & $\begin{array}{r}\text { Gal. lat. } \\
\text { (deg) }\end{array}$ & $\begin{array}{r}\text { ISO-LWS [CII] } \\
\left(\mathrm{W} \mathrm{cm}^{-2} \times 10^{-19}\right)\end{array}$ & 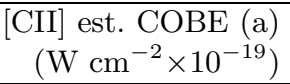 & $\begin{array}{r}\text { [CII] cor. value } \\
\left(\mathrm{W} \mathrm{cm}^{-2} \times 10^{-19}\right)\end{array}$ & $\begin{array}{l}\text { [CII] upper limit (b) } \\
\left(\mathrm{W} \mathrm{cm}^{-2} \times 10^{-19}\right)\end{array}$ \\
\hline AA Tau & -15.40 & $1.13 \pm 0.10$ & 0.64 & $0.49 \pm 0.10$ & $\ldots$ \\
\hline GG Tau & -20.25 & $0.59 \pm 0.09$ & 0.49 & $0.10 \pm 0.09$ & $\ldots$ \\
\hline AB Aur & -7.98 & $0.71 \pm 0.07$ & $\ldots$ & $\ldots$ & $\leq 0.71$ \\
\hline CQ Tau & -26.17 & $1.73 \pm 0.07$ & 0.39 & $1.34 \pm 0.07$ & $\ldots$ \\
\hline HD 36112 & -25.95 & $1.08 \pm 0.01$ & 0.39 & $0.69 \pm 0.10$ & $\ldots$ \\
\hline $\mathrm{LkH} \alpha 233$ & -15.14 & $4.63 \pm 0.20$ & 0.65 & $3.98 \pm 0.20$ & $\ldots$ \\
\hline MWC 340 & +2.77 & $57.00 \pm 0.86$ & $\ldots$ & $\ldots$ & $\leq 57.00$ \\
\hline MWC 480 & -7.90 & $0.59 \pm 0.06$ & $\ldots$ & $\ldots$ & $\leq 0.59$ \\
\hline
\end{tabular}

a - Based on Bennett et al. (1994).

b - For those stars for which we were unable to use Bennett et al.'s COBE estimation for the [CII] background, the ISO-LWS measurement serves as an upper limit to the [CII] flux.

predictions of Kaufman's and previous PDR models (e.g. Hollenbach et al. 1991; Wolfire et al. 1990) stem from the inclusion of heating due to the ejection of photoelectrons from PAHs and small dust grains, and from the use of lower carbon and oxygen abundances. It has been observationally shown that PAHs and dust are an essential part of the YSO environment (e.g. Dent et al. 1998; Hanner et al. 1998; Siebenmorgen et al. 2000). Kaufman et al. demonstrate that the addition of this heating source lowers the values of both density and radiation field flux derived, as compared to previous PDR models which do not include very small grains and PAHs.

Based on the predictions of shock models, we have ascertained the dominant excitation mechanism for the fine-structure lines seen in our sample to be that from stellar ultraviolet photons rather than shocks. Our conclusions are based on arguments similar to those found in Lorenzetti et al. (1999) and we direct the reader's attention there for a full discussion. In short, J-shock excitation, such as that modelled by Hollenbach \& McKee (1989), can be ruled out, since such models predict line ratios of 30-200 for [OI]63/[OI]145 and $10^{1-4}$ for [OI]63/[CII]158. Alternatively, the $C$-shock or "cool PDR" models of Draine et al. (1983) predict no substantial [CII] emission.

Figure 3 shows that the observed ratios for $\mathrm{LkH} \alpha 233$, CQ Tau, and to a lesser extent MWC 480 and AB Aur are not inconsistent with the PDR models for moderate intensities of the radiation field $\left(G_{\mathrm{O}}<10^{4}\right)$, appropriate for the young Herbig Ae stars illuminating their environments. Similarly, comparison of the observed [O I] $63 \mu \mathrm{m} /[\mathrm{C} \mathrm{II}]$ $158 \mu \mathrm{m}$ flux ratios with Fig. 4 of Kaufman et al. (1999) points toward a high-density $\left(>10^{3} \mathrm{~cm}^{-3}\right)$, moderate $G_{\mathrm{o}}$ $\left(10^{2}-10^{3}\right)$ regime. Higher angular resolution observations are needed to separate the disk from the envelope emission. Some [C II] emission is predicted from the warm upper layers of disks, where CO can be photodissociated and $\mathrm{C}$ photoionized by the interstellar radiation field (e.g., Aikawa \& Herbst 1999; Willacy \& Langer 2000).

\subsection{Molecular transitions}

In general, few or no molecular transitions were found that were deemed veritable in seven of the eight stars examined here. We believe this is due to the general unembedded nature of these sources, and the lack of sensitivity of ISOLWS for these fainter sources in these relatively rapid scans. However, we do see a plethora of CO lines in the most deeply embedded object in this sample, MWC 340, all in agreement with van den Ancker et al. (2000) at the $30 \%$ level. These CO lines seen in the MWC 340 spectra originate in the warm, dense envelope surrounding the young star. In order to aid the astrophysical community in modelling the environments of YSOs, we report a $3 \sigma$ rms flux level at the line center positions for some astrophysically important transitions of $\mathrm{CO}, \mathrm{H}_{2} \mathrm{O}$ and $\mathrm{OH}$ (Table 3). It will be necessary in future missions to make these measurements using smaller beams if molecular lines are to be detected from the disks of these YSOs.

\section{SED modelling}

\subsection{Chiang \& Goldreich models}

Chiang \& Goldreich (1997, hereafter CG97) compute spectral energy distributions (SEDs) of passive disks in radiative and hydrostatic balance with their central isolated stars. The passive disk divides naturally into two regions: a surface layer that contains dust grains directly exposed to central starlight, and a cooler interior that is encased and diffusively heated by the surface. Dust grains in the surface layer reradiate to space about half the stellar energy they absorb. The other half is emitted towards the midplane and regulates the temperature of the interior. The disk surface flares outward with increasing radius and it intercepts more stellar radiation than a flat disk would, especially at distances for which the height of the disk exceeds the stellar radius.

The models of Chiang et al. (2001, hereafter C01) improve upon those of CG97 by (1) accounting for a range 

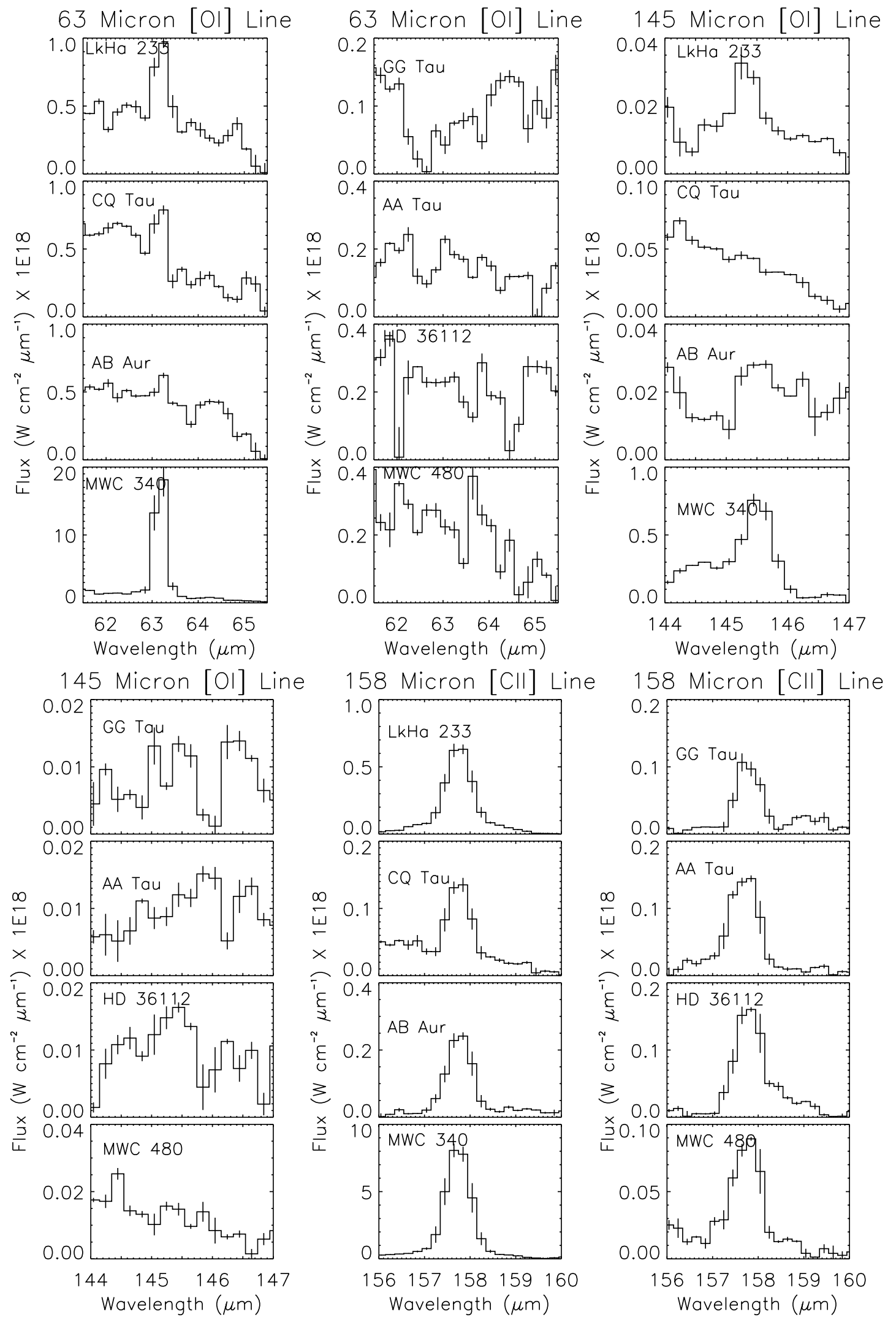

Fig. 2. Shown here are histogram plots of the three principal fine-structure lines used in the PDR modelling (63 $\mu \mathrm{m}[\mathrm{OI}]$, $145 \mu \mathrm{m}[\mathrm{OI}]$ and $158 \mu \mathrm{m}[\mathrm{CII}])$ for the sources. In all cases, a constant has been subtracted from the data to render them on similar scales. However, no slope or fitted baseline has been used. Both fine-structure transitions of [OI] are evident in only a few sources. In cases where a line is not evident, the $3 \sigma$ baseline noise values are instead quoted in Table 2 . While the $158 \mu \mathrm{m}$ line is clearly evident in all sources, its presence is not strictly indicative of [CII] transitions in the YSO environment itself. For the PDR modelling, a correction to this line strength is made based upon COBE data (see text for details). 


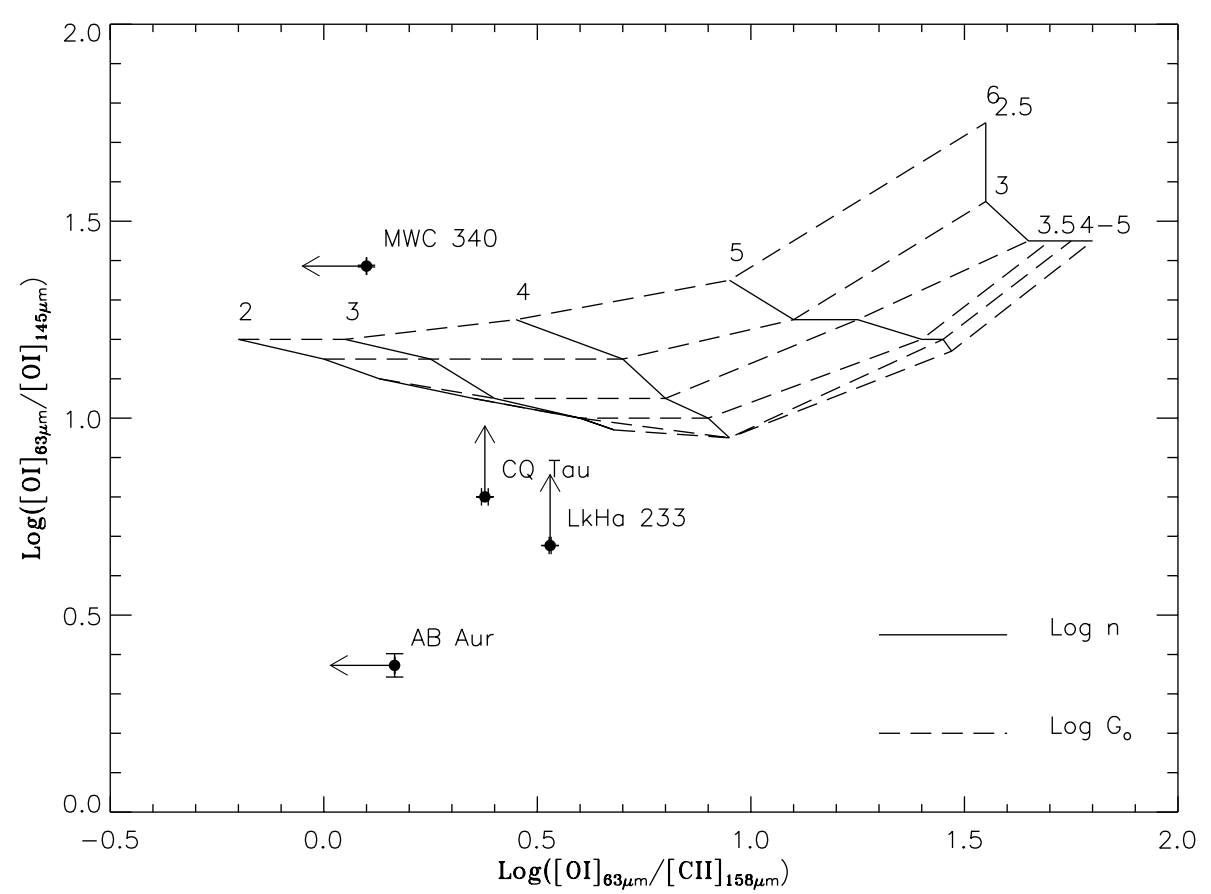

Fig. 3. Observed ratios of fine-structure lines plotted on the PDR Models of Kaufman et al. (1999). One-sigma error bars are included, and arrows to indicate uncertainties when a line was not identified at the $3 \sigma$ level.

of particle sizes, (2) employing laboratory-based optical constants of water ice, olivine, and iron to compute the emissive properties and temperatures of disk grains, and (3) solving numerically the equations of radiative and hydrostatic equilibrium under the 2-layer approximation of CG97. These improvements are motivated largely by the new high-resolution ISO data presented herein.

We employ the flared, passive disk models of C01 to fit ISO-LWS observations of the subset of eligible systems in our sample: objects that are (1) not known binaries or known to be confused in the arcminute-sized ISO beam, and (2) not known to drive jets or to be surrounded by large ( $2500 \mathrm{AU}$-scale) nebulosities that are better described by spherical envelopes rather than flattened disks. In order of increasing stellar effective temperature, the eligible stars are AA Tau, CQ Tau (HD 36910), HD 36112 (MWC 758), and MWC 480 (HD 31648). Note that we have omitted $\mathrm{AB}$ Aur from this list; this system contains a tenuous, large-scale envelope which has been argued to substantially heat an embedded circumstellar disk (Miroshnichenko et al. 1999; Grady et al. 1999). We further note that Bouwman et al. (2000) argue for two distinct temperature distribution regions in this system to explain its SED. These fitted model spectra are meant only to be illustrative and to enable a first-order description of the nature of these sources.

We refer the reader to CG97 and C01 for a more pedagogical exposition of the input parameters and physics underlying their models. C01 also contains some comparitive discussions regarding other models in the literature. Here we simply outline the principal ingredients of the new C01 models that are not contained in CG97. Where local dust temperatures (=gas temperatures) fall below $T_{\text {sub }}^{\text {ice }} \approx 150 \mathrm{~K}$, the grains are taken to be spheres of amorphous olivine mantled with water ice. For simplicity, the thickness of the water ice mantle relative to the radius of the olivine core is held constant. Where local temperatures fall between $T_{\text {sub }}^{\text {ice }}$ and $T_{\text {sub }}^{\text {sil }} \approx 1500 \mathrm{~K}$, only the pure olivine cores are assumed to remain. In innermost disk regions where local temperatures fall between $T_{\text {sub }}^{\text {sil }}$ and $T_{\mathrm{sub}}^{\mathrm{iron}} \approx 2000 \mathrm{~K}$, the grains are taken to be spheres of metallic iron.

The iron or silicate cores in the disk surface s (interior i) possess a power law distribution of radii $r$ between $r_{\min }$ and $r_{\max , \mathrm{s}}\left(r_{\max , \mathrm{i}}\right)$ :

$\mathrm{d} N \propto r^{-q_{\mathrm{i}(\mathrm{s})}} \mathrm{d} r$

where $\mathrm{d} N$ is the number density of grains having radii between $r$ and $r+\mathrm{d} r$. In practice, $r_{\min }$ is fixed at $10^{-2} \mu \mathrm{m}$, while $r_{\max , \mathrm{i}}, r_{\max , \mathrm{s}}, q_{\mathrm{i}}$, and $q_{\mathrm{s}}$ are free to vary. Generally $r_{\text {max }, \mathrm{s}}<r_{\text {max }, \mathrm{i}}$ since large grains tend to settle quickly out of tenuous surface layers (see Sect. 3.3 of CG97). All of the cosmically abundant iron is assumed to be locked within grains. Following Pollack et al. (1994), we take $50 \%$ of the cosmically abundant oxygen to be locked in $\mathrm{H}_{2} \mathrm{O}$ ice. Together, these assumptions yield a fractional thickness, $\Delta r / r$, for the water ice mantle equal to 0.5 .

Optical constants for amorphous olivine are obtained from the University of Jena Database (http://www.astro.uni-jena.de; see also Jäger et al. 1994). Longward of $500 \mu \mathrm{m}$ where such data for silicates are not available, the complex refractive index $(n+i k)$ for glassy olivine is extrapolated such that $n(\lambda \geq 500 \mu \mathrm{m})=n(500 \mu \mathrm{m})$ and $k(\lambda \geq 500 \mu \mathrm{m})=k(500 \mu \mathrm{m})\left(\frac{\lambda}{500 \mu \mathrm{m}}\right)^{-1.01}$. Optical 


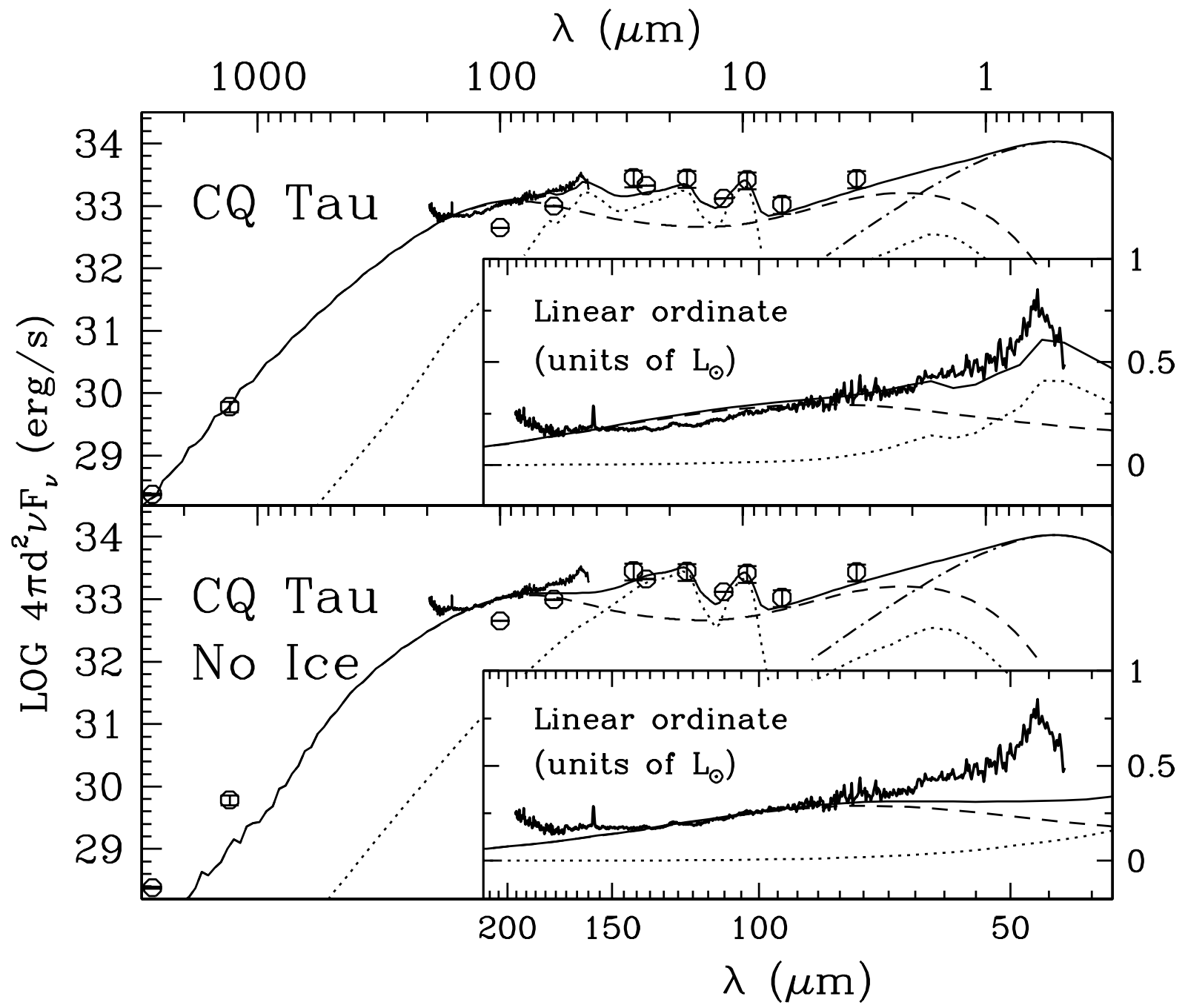

Fig. 4. C01 passive disk model fitted to CQ Tau with and without ice. The dot-dashed line indicates the stellar contribution, the dashed line the contribution from the disk interior, and the dotted line the contribution from the irradiated disk surface. The solid line denotes the total SED. The heavier solid line (from 43 to $195 \mu \mathrm{m}$ ) is our ISO-LWS spectrum. Open circles represent ground and space-based archival data. The inset plot with linear ordinate magnifies the spectral region spanned by ISO LWS. We note that not only are the emission feaures in the ISO-LWS data not reproduced without the inclusion of ice, but the entire mm-wave continuum fails to be modelled by pure silicate grains alone. See text for full discussion.

constants for pure crystalline $\mathrm{H}_{2} \mathrm{O}$ ice are taken from the NASA ftp site (ftp:climate.gsfc.nasa.gov/pub/ wiscombe/Refrac_Index/ICE/; see also Warren 1984). Optical constants for metallic $\mathrm{Fe}$ are obtained from Pollack et al. (1994).

Central stars are modelled as blackbodies whose effective temperatures and radii are taken from the literature; references are provided in footnote (a) to Table 5. Visual extinctions range from $A_{V}=0.3 \mathrm{mag}$ (MWC 480) to $1.6 \mathrm{mag}$ (CQ Tau). These modest values imply that the central stars are not significantly occulted by the flared outer edges of their disks and that the simplifying assumption of a face-on viewing geometry is adequate for computation of the SEDs. As demonstrated by Chiang \& Goldreich (1999), the SED varies negligibly with inclination over viewing angles for which the flared disk does not occult the central star because the infrared fluxes emerge mainly from optically thin surface layers while the mm-wave emission originates mostly from the optically thin interior.

\subsection{Model fits}

\subsubsection{Continuum}

Observed and theoretical SEDs are compared in Figs. 4-5. For each source, a model SED is fitted to the ISO-LWS scan, millimeter wavelength fluxes, and $\sim 5-25 \mu \mathrm{m}$ photometric data. Meanings and values of fitted parameters are listed in Table 5, and are used henceforth without explanation.

For all stars in the sample, agreement between the theoretical continuum and the observed ISO-LWS continuum is often good to well within a factor of 2 . The bulk of the flux at ISO-LWS wavelengths emerges from the cooler, diffusively heated disk interior at large radius. 
Table 5. Model parameters ${ }^{a}$.

\begin{tabular}{|c|c|c|c|c|c|}
\hline Parameter & Meaning & MWC 480 & HD 36112 & CQ Tau & AA Tau \\
\hline$T_{*}(\mathrm{~K})$ & Stellar effective temperature ${ }^{b}$ & 8890 & 8465 & 7130 & 4000 \\
\hline$R_{*}\left(R_{\odot}\right)$ & Stellar blackbody radius ${ }^{b}$ & 2.1 & 2.1 & 1.9 & 2.1 \\
\hline$M_{*}\left(M_{\odot}\right)$ & Stellar mass ${ }^{b}$ & 2.3 & 2.2 & 1.7 & 0.67 \\
\hline$d(\mathrm{pc})$ & Source distance ${ }^{b}$ & 140 & 150 & 150 & 140 \\
\hline$\Sigma_{0}=\Sigma a_{\mathrm{AU}}^{3 / 2}\left(\mathrm{~g} \mathrm{~cm}^{-2}\right)$ & Disk surface density $^{c}$ at $1 \mathrm{AU}$ & 8000 & 1000 & 3000 & 1500 \\
\hline$a_{\mathrm{o}}\left(10^{4} R_{*}\right)[\mathrm{AU}]$ & Outer disk truncation radius ${ }^{c}$ & $1.1[100]$ & $2.5[250]$ & $2.8[250]$ & $2.6[250]$ \\
\hline$H / h$ & Height of visible photosphere in scale heights ${ }^{d}$ & 1.7 & 1.5 & 4.7 & 3.8 \\
\hline$q_{i}$ & Grain size index ${ }^{c}$ in interior & 2.8 & 3.5 & 3.0 & 3.5 \\
\hline$r_{\max , i}(\mu \mathrm{m})$ & Maximum grain size ${ }^{c}$ in interior & 1000 & 1000 & 1000 & 1000 \\
\hline$M_{\mathrm{DISK}}\left(M_{\odot}\right)$ & Total disk $\operatorname{mass}^{e}$ (gas and dust) & 0.11 & 0.02 & 0.07 & 0.03 \\
\hline$H\left(a_{\mathrm{o}}\right) / a_{\mathrm{o}}$ & 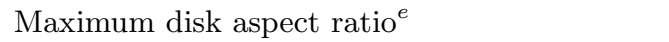 & 0.13 & 0.16 & 0.54 & 0.58 \\
\hline
\end{tabular}

${ }^{a}$ For all sources, we fix $q_{\mathrm{s}}=3.5$ (grain size index in surface), $r_{\max , \mathrm{s}}=1 \mu \mathrm{m}$ (maximum grain size in surface), and $a_{i}=2 R_{*}$ (inner disk truncation radius). See C01 for rationale.

${ }^{b}$ Stellar parameters and distances of Herbig AeBe stars are taken from Mannings \& Sargent (1997). For T Tauri star AA Tau, stellar parameters are taken from Beckwith et al. (1990).

${ }^{c}$ The continuum SED is largely degenerate with respect to simultaneous changes in $\Sigma_{0}, r_{\max , \mathrm{i}}, q_{\mathrm{i}}$, and $a_{\mathrm{o}}$. The values shown here are not uniquely constrained. See C01 for discussion.

${ }^{d}$ This is the one disk parameter which appears most uniquely constrained by the SED alone. Values less than $4-5$ imply significant vertical settling of photospheric dust grains.

${ }^{e}$ Total masses and maximum aspect ratios of fitted disks are derived quantities and not input parameters. As discussed in $\mathrm{C} 01$, the total disk mass depends sensitively on the a priori unknown millimeter-wave opacity and could vary by an order of magnitude.

Depending upon the source, the luminosity emerging at ISO-LWS wavelengths equals $\sim 1-10 \%$ of $L_{*}$ (central stellar luminosity). Passive, flared disks account naturally for the magnitude of this far-infrared luminosity. As shown in CG97,

$4 \pi d^{2} \nu F_{\nu} \sim\left[\left(\frac{\mathrm{d} \ln H}{\mathrm{~d} \ln a}-1\right) \frac{H}{a}+\frac{R_{*}}{a}\right] \frac{L_{*}}{2}$

where $H$ is the vertical height of the visible photosphere above the disk midplane, $a$ is the radial distance to the disk rotation axis in cylindrical coordinates, and all other variables have their usual meaning. We note that the quantity in square brackets equals the angle at which central stellar radiation strikes the visible photosphere of the disk, as measured from the tangent plane of the disk surface. At ISO-LWS wavelengths, this incident grazing angle is to be evaluated near the outer truncation radius of the disk, $a \approx a_{\mathrm{o}} \approx O\left(10^{4} R_{*}\right)$. The fitted outer truncation radii listed in Table 5 are in accord with upper limits set by Mannings \& Sargent (1997) and Dutrey et al. (1996) using interferometric imaging at $\lambda=2.7 \mathrm{~mm}$. Typically, the curvature of the disk surface is such that $\mathrm{d} \ln H / \mathrm{d} \ln a-1 \approx 0.2$ (CG97, C01) and the maximum aspect ratio of the disk is $H / a_{\mathrm{o}} \approx 0.3$ (see Table 5). Inserting these values into Eq. (3), we find that $4 \pi d^{2} \nu F_{\nu} \sim$ $0.03 L_{*}$, in order-of-magnitude agreement with the observed ISO continuum. If the passive disk were modelled (in neglect of hydrostatic balance) as infinitesimally flat or as a wedge (constant opening angle), the grazing angle reduces to $R_{*} / a_{\mathrm{o}} \approx O\left(10^{-4}\right)$, and predicted fluxes would be too low compared to observations by about 2 orders of magnitude. Hydrostatic flaring ensures that the disk intercepts sufficient stellar radiation to explain the magnitude of the ISO-LWS fluxes.

Note that for MWC 480, HD 36112 and AA Tau (cf. Table 5), the number of gas scale heights that the visible photosphere sits above the disk midplane, $H / h$, is set to values less than 4 . If gas and dust were well-mixed in interstellar proportions, then $H / h \approx 4-5$ (CG97). Lower values correspond to more flattened disks and are required for fits to these stars so that model SEDs do not exceed the observed fluxes. Such low values for $H / h$ may indicate downward gravitational settling of photospheric, superheated dust through the uppermost, tenuous gas layers. However, recent papers also suggest that these low $H / h$ values could also be explained by a disk rim (Natta et al. 2001; Dullemond et al. 2001). In a quiescent nebula, the timescale for a dust grain of size $r$ and mass density $\rho_{\mathrm{p}}=2 \mathrm{~g} \mathrm{~cm}^{-3}$ to settle from height $z=4 h$ to $z=1 h$ is

$$
\begin{aligned}
t_{\text {settle }} \approx 0.1 \frac{\Sigma}{\rho_{\mathrm{p}} r \Omega} \approx & 10^{5}\left(\frac{0.1 \mu \mathrm{m}}{r}\right) \\
& \times\left(\frac{\Sigma_{0}}{300 \mathrm{~g} \mathrm{~cm}^{-2}}\right)\left(\frac{2 M_{\odot}}{M_{*}}\right)^{1 / 2} \mathrm{yr},
\end{aligned}
$$

where $\Omega$ is the local orbital angular frequency of disk material. The settling time for a disk with our assumed surface density profile is independent of $a$ and well within the estimated Myr ages of these stars. The actual amount of settling also depends upon the unknown degree of turbulence and vertical circulation in nebular gas. As a final note, it is important to point out that for the weakest 
sources, such as AA Tau, it is possible to have additional emission in the beam longward of $150 \mu \mathrm{m}$ due to cirrus emission, and that this too can change the apparent farinfrared SED.

\subsubsection{Solid state features from water ice and crystalline silicates}

Passive disks exhibit infrared emission features associated with dust grain resonances. Solid state emission features arise from line-of-sight material which is optically thin in the adjacent continuum. Such material can originate from superheated surface layers and/or the optically thin disk interior.

Longward of $\sim 40 \mu \mathrm{m}$, optically active modes of vibration in crystalline water ice (or crystalline silicates) are "intermolecular translational" or "intermolecular librational", involving collective movement of molecules or a unit cell with respect to other molecules/unit cells in the lattice. These modes are more sensitive to the presence of chemical impurities and to long-range order in the solid than fundamental $\mathrm{O}-\mathrm{H}$ and $\mathrm{O}-\mathrm{H}-\mathrm{O}$ stretching and bending modes at shorter wavelengths. As noted in Sect. 4.1, intermolecular modes provide important evidence for the annealing of amorphous interstellar silicates and ices in the high density/high temperature disk environment, and may provide clues as to how grains formed in the ISM are transformed into the materials prevalent in the early solar nebula. Translational modes in pure, ordinary hexagonal $\mathrm{H}_{2} \mathrm{O}$ ice (type Ih) lie at $\sim 45,62,100$, and $154 \mu \mathrm{m}$ (Bertie et al. 1969, see their Figs. 4 and 11).

The coolest stars in our subsample, AA Tau and CQ Tau, evince solid state emission features in their ISO-LWS scans associated with water ice. In CQ Tau (Fig. 4), we identify the strongest peak at $46.5 \mu \mathrm{m}$ with the translational mode in water ice having the highest oscillator strength. We take this same resonance to be partly responsible for the sharp rise in flux shortward of $50 \mu \mathrm{m}$ in AA Tau (Fig. 5). Though more noisy, the lattice mode at $62 \mu \mathrm{m}$ may also be in emission in both stars. Water ice emission at these wavelengths arises largely from icy grains in superheated surface layers located at outermost disk radii ( $a \gtrsim 100 \mathrm{AU}$ ). For both CQ Tau and AA Tau, the disk interior at LWS wavelengths is optically thick and contributes only a featureless continuum.

To highlight the need for water ice in fitting the SED of CQ Tau, we attempt to fit a second disk model without water ice to the observed spectrum in Fig. 4 . The fit is clearly much poorer if water ice does not coat the grains; not only are emission features in the ISO LWS data not reproduced, but the entire millimeter-wave continuum fails to be modelled by pure silicate grains alone. The fit in Fig. 4 is the best of many trial fits, and corresponds to a $0.20 M_{\text {sol }}$ disk; we could increase the disk mass further to try to achieve a better match to the millimeter-wave continuum (while still failing to fit the ISO-LWS scan), but such a disk would be gravitationally unstable. We also find that no satisfactory fit can be obtained by varying the grain size distribution. Thus, we consider the interpretation that water ice coats the grains in CQ Tau to be secure.

The hotter stars, HD 36112 and MWC 480, do not evince water ice emission at $46 \mu \mathrm{m}$. This may simply reflect decreasing amounts of water ice in the disk surface with increasing stellar/disk temperatures. The ice condensation boundary in the optically thin disk surface moves outward approximately as $T_{*}^{3}(\mathrm{C} 01)$. It is tempting to identify ice emission bands at $62 \mu \mathrm{m}$ for these two stars, but then the absence of the $45 \mu \mathrm{m}$ band, which possesses the higher oscillator strength, becomes problematic.

Our fits to the ISO-LWS spectra clearly require improvement. Accurate reproduction of translational water ice bands is hampered by a number of entangled difficulties, including (1) uncertainties in the photospheric abundances of water relative to silicates; (2) how ice is distributed with particle size; (3) the probable presence of impurities in water ice that can shift band positions and widths; (4) incompleteness of laboratory data for the optical constants of a cosmic mixture of ices in various allotropic states at wavelengths longward of $100 \mu \mathrm{m}$; and (5) possibility of instrumental error. We refer the reader to C01 for a complete discussion of issues 1-4.

With regards to point (5) above, known filter leaks, caused by near-infrared radiation contaminating the higher order wavebands of LWS, generate spurious emission features of widths $1.8-4.3 \mu \mathrm{m}$ at $53.6,105.1,109.3$, and possibly 51 and $57 \mu \mathrm{m}$ in spectra of sources brighter than $H=2.2 \mathrm{mag}^{2}$. While none of the sources presented herein meet the criteria outlined in the ISO-LWS report on the near-infrared leak, it is worth noting that some spectral features may be artificial.

Silicate emission bands from the asymmetric $\mathrm{Si}-\mathrm{O}$ stretching mode at $10 \mu \mathrm{m}$ appear in CQ Tau, HD 36112 and MWC 480 in the literature; there are no observations of AA Tau with which to determine the presence/absence of such a feature. These bands are naturally modelled as emission from optically thin, superheated disk atmospheres. In MWC 480 , the $10 \mu \mathrm{m}$ resonance appears as a resolved emission line in spectra from the Kuiper Airborne Observatory (Sitko et al. 1999 their Fig. 1). Its shape is imperfectly fitted by our model, indicating that actual surface layer silicates in this source have allotropic states (crystalline vs. amorphous) and compositions (pyroxene vs. olivine, and Fe:Mg ratios) slightly different from the amorphous $\mathrm{MgFeSiO}_{4}$ that we employ.

Evidence for crystalline silicates may also exist in the ISO-LWS scans. In Mg-rich silicates, such as forsterite or natural olivine, modes are predominantly translational longward of $\sim 25 \mu \mathrm{m}$ (Jäger et al. 1998). A broad emission feature at $80 \mu \mathrm{m}$ is present in scans of $\mathrm{AA}$ Tau, MWC 480, and possibly CQ Tau and HD 36112. No resonance is measured at $80 \mu \mathrm{m}$ for the crystalline

\footnotetext{
2 See http://www.iso.vilspa.esa.es/notes/ lws_0197.html for complete details.
} 


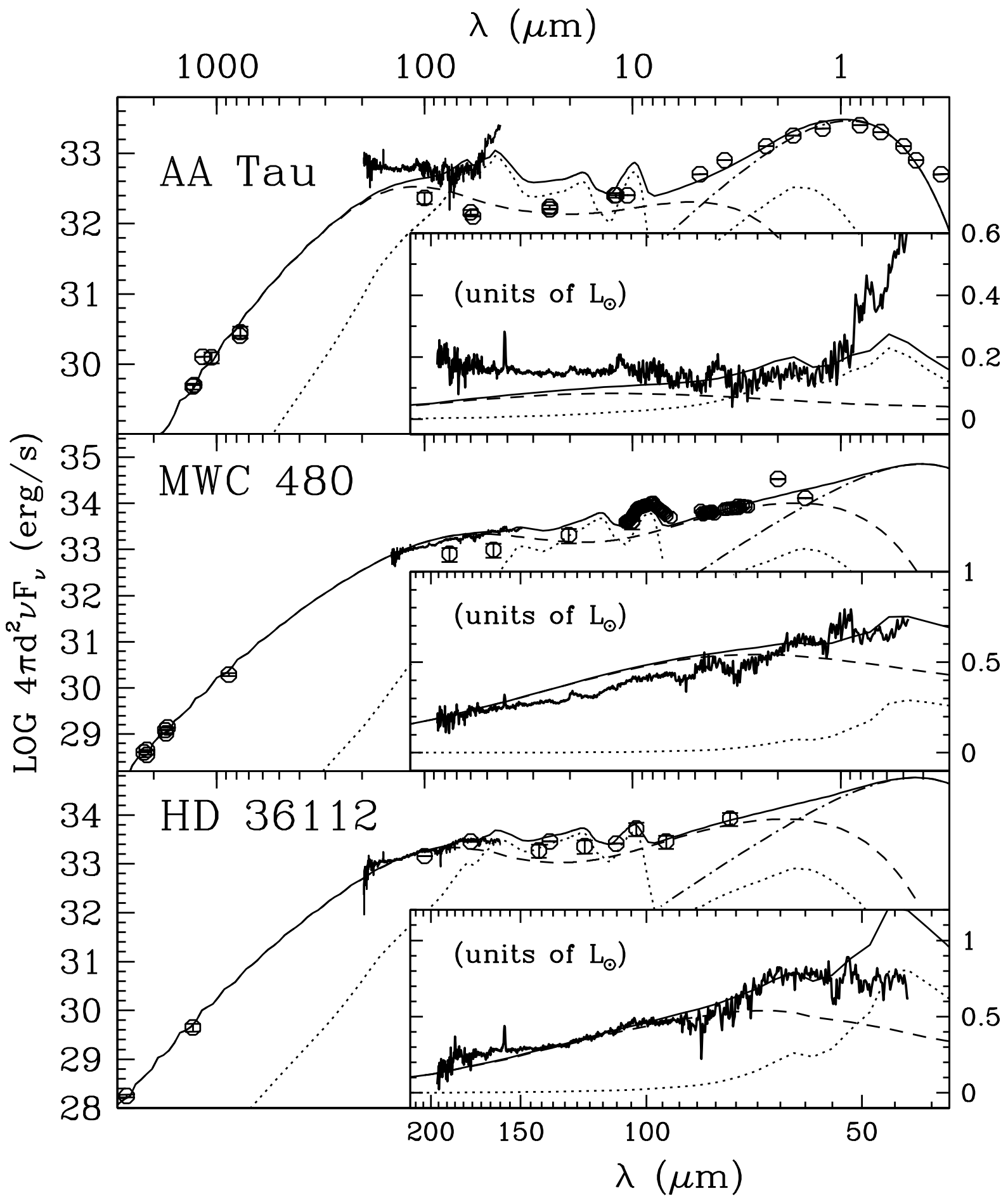

Fig. 5. Chiang et al. (2001) passive disk model (with ice) fitted to data for AA Tau, MWC 480 and HD 36112. See caption for Fig. 4.

silicates examined by Jäger et al. (1998). However, these authors also show that peak positions of a given vibrational mode shift towards longer wavelengths with increasing iron-to-magnesium content (higher effective vibrating masses). We propose that the $80 \mu \mathrm{m}$ feature is caused by a translational mode in a crystalline silicate having an $\mathrm{Fe} / \mathrm{Mg}$ ratio intermediate between that of natural olivine $\left(\mathrm{Mg}_{1.96} \mathrm{Fe}_{0.04} \mathrm{SiO}_{4}\right)$ and natural hortonolite
$\left(\mathrm{Mg}_{1.1} \mathrm{Fe}_{0.9} \mathrm{SiO}_{4}\right)$ (Jäger et al. 1998, see their Table 3). If this is the case, we would expect another silicate translational mode to appear in emission near $51 \mu \mathrm{m}$; indeed, such an emission feature does appear in ISO scans of AA Tau, MWC 480, and HD 36112. An unidentified, solidstate emission feature is present at $109 \mu \mathrm{m}$ in the AA Tau spectrum. We speculate that it may also arise from translational modes in crystalline silicates. (Although we do 
again note that this is where one of the ISO-LWS nearinfrared leaks is seen in bright sources.) To date, mineral spectroscopy in the laboratory rarely extends beyond $100 \mu \mathrm{m}$.

\section{Conclusions}

The ISO-LWS spectra presented here of eight YSOs from a variety of spectral and evolutionary stages exhibit an interesting mix of spectral lines and SEDs. In particular, we can make the following conclusions based on the data presented herein.

- Fine structure lines of [O I] and/or [C II] are detected for all our sources. For the embedded objects, we have ascertained that the dominant mechanism for the production of these lines is ultraviolet stellar photons, rather than shocks. Higher angular resolution observations are needed to observe fine-structure and molecular lines from circumstellar disks.

- Isolated, flared, passively heated circumstellar disks adequately explain ISO-LWS data for four of our sample stars. The bulk of the flux at LWS wavelengths emerges from the cooler, diffusively heated disk interior at stellocentric distances $\gtrsim 100 \mathrm{AU}$. Water ice emission bands appear in the ISO-LWS scans of the two coolest stars, CQ Tau and AA Tau, but are absent in ISO-LWS scans of the hotter stars, MWC 480 and HD 36112. Silicate emission bands appear in nearly all sources. These solid-state emission bands arise naturally from icy silicate grains in optically thin disk surface layers.

Acknowledgements. We would like to graciously acknowledge the ESA, NASA and the ISO-LWS instrument team and reduction software groups in Vilspa and Pasadena for all their work and generous support of the ISO Mission. M.J.C-E. in particular gratefully acknowledges the assistance of T. Lim, S. Molinari and S. Lord in reduction and quality control of the ISO data. We would also like to thank T. Prusti for useful and insightful comments, particularly with regard to the background contributions to the dataset. G.A.B. acknowledges support from the ISO block grant program and NASA grant NAG5-4303. E.F.vD. acknowledges support from NWO grant 614.41.003. E.I.C. acknowledges support from NASA through grant NAG5-7008 and a Hubble Fellowship grant awarded by the Space Telescope Science Institute, which is operated by the Association of Universities for Research in Astronomy, Inc., for NASA under contract NAS 5-26555. We also wish to thank M. van den Ancker, R. Waters, J. Bouwman and D. Lorenzetti for useful discussions and communicating their results prior to publication. We have made use of the COBE and IRAS databases maintained through GSFC and IPAC at Caltech as part of this study. We have also made use of the SIMBAD database created and maintained by the CDS, Strasbourg and ADS Abstract Service created and maintained by the CFA, Harvard in preparation of this manuscript.

\section{References}

Adams, F. C., \& Lin, D. N. C. 1993, in Protostars \& Planets III, ed. E. H. Levy, \& J. Lunine (Univ. Arizona Press, Tucson), 721

Aikawa, Y., \& Herbst, E. 1999, A\&A, 351, 233

Beckwith, S. V. W., Sargent, A. I., Chini, R. S. \& Güsten, R. 1990, AJ, 99, 924

Beckwith, S. V. W., \& Sargent, A. I. 1996, Nature, 383, 139

Bennett, C. L., Fixsen, D. J., Hinshaw, G., et al., 1994, ApJ, 434,587

Bertie, J. E., Labbé, H. J., \& Whalley, E. 1969, J. Chem. Phys., 50,4501

Bock, J. J., Hristov, V. V., Kawada, M., et al. 1993, ApJ, 410, $\mathrm{L} 115$

Bouwman, J., de Koter, A., van den Ancker, M. E., \& Waters, L. B. F. M. 2000, A\&A, 360, 213

Burrows, C. J., Stapelfeldt, K. R., Watson, K. R., et al. 1996, ApJ, 473, 437

Ceccarelli, C., Haas, M. R., Hollenbach, D. J. \& Rudolph, A. L. 1997, ApJ, 476, 771

Chiang, E. I., \& Goldreich, P. 1997, ApJ, 490, 368 (CG97)

Chiang, E. I., \& Goldreich, P. 1999, ApJ, 519, 279

Chiang, E. I., Joung, M. K., Greech-Eakman, M. J., et al. 2001, ApJ, 547, 1077 (C01)

Clegg, P., et al. 1996, A\&A, 315, 38

Dent, W. R. F, Matthews, H. E., \& Ward-Thompson, D. 1998, MNRAS, 301, 1049

Draine, B. T., Roberge, W. G., \& Dalgarno, A. 1983, ApJ, 264, 485

Dullemond, C. P., Dominik, C., \& Natta, A. 2001, ApJ, 560, 957

Dutrey, A., Guilloteau, S., Duvert, G., et al. 1996, A\&A, 309, 493

Giannini, T., Lorenzetti, D., Tommasi, E., et al. 1999, A\&A, 346,617

Grady, C. A., Woodgate, B., Bruhweiler, F. C., et al. 1999, ApJ, 523, L151

Guilloteau, S., \& Dutrey, A. 1998, A\&A, 339, 467

Hanner, M. S., Brooke, T. Y., \& Tokunaga, A. T. 1998, ApJ, 502,871

Hollenbach, D. J., \& McKee, C. F. 1989, ApJ, 342, 306

Hollenbach, D. J., Tielens, A. G. G. M., \& Takahashi, T. 1991, ApJ, 377, 192

Jäger, C., Molster, F. J., Dorschner, J., et al. 1998, A\&A, 339, 904

Jäger, C., Mutschke, H., Begemann, B., Dorschner, J., \& Henning, Th. 1994, A\&A, 292, 641

Kaufman, M. J., Wolfire, M. G., Hollenbach, D. J., \& Luhman, M. L. 1999, ApJ , 527, 795

Kenyon, S. J., Dobrzycka, D., \& Hartmann, L. 1994, AJ, 108, 1872

Kessler, Steinz, J. A., Anderegg, M. E., et al. 1996, A\&A, 315, 27

Koerner, D. W., \& Sargent, A. I. 1995, AJ, 109, 2138

Koresko, C. 1998, ApJ, 507, L145

Lada, C. J. 1991, in The Physics of Star Formation and Early Stellar Evolution, ed. C. J. Lada, \& N. D. Kylafis (Kluwer, Dordrecht), 329

Langer, W. D., et al. 2000, in Protostars and Planets IV, ed. V. Mannings, A. P. Boss, \& S. S. Russell (Univ. of Arizona Press), 29

Li, Z.-Y., \& Shu, F. H. 1996, ApJ, 468, 261

Lorenzetti, D. 1998, priv. comm. 
Lorenzetti, D., et al. 1999, A\&A, 346, 604

Malfait, K., Waelkens, C., Waters, L. B. F. M., et al. 1998, A\&A, 332, L25

Marcy, G. W., \& Bulter, R. P. 1996, ApJ, 464, L147

Mannings, V., \& Sargent, A. I. 1997, ApJ, 490, 792

McCaughrean, M. J., Stapelfeldt, K. R., \& Close, L. M. 2000, in Protostars and Planets IV, ed. V. Mannings, A. P. Boss, \& S. S. Russell (Univ. of Arizona Press), 485

Miroshnichenko, A., Ivezić, Z., Vinković, D., \& Elitzur, M. 1999, ApJ, 520, L115

Mundy, L. G., Looney, L. W. \& Welch, W. J. 2000, in Protostars and Planets IV, ed. V. Mannings, A. P. Boss, \& S. S. Russell (Univ. of Arizona Press), 355

Natta, A., Prusti, T., Neri, R., et al. 2001, A\&A, 371, 186

Nisini, B., Lorenzetti, D., Cohen, M., et al. 1996, A\&A, 315, 321

Pollack, J. B., Hollenback, D., Beckwith, S., et al. 1994, ApJ , 421,615

Siebenmorgen, R., Prusti, T., Natta, A., \& Müller, T. G. 2000, A\&A, 361, 258

Sitko, M. L., Grady, C. A., Lynch, D. K., Russell, R. W., \& Hanner, M. S. 1999, ApJ, 510, 408
Stark, R. 1998, priv. comm.

Swinyard, B. M., Clegg, P. E., Ade, P. A. R., et al. 1996, A\&A, 315,43

Testi, L., Natta, A, Shepherd, D. S., \& Wilner, D. J. 2001, ApJ, 554, 1087

Thé, P. S., de Winter, D., \& Pérez, M. R. 1994, A\&AS, 104, 315

van den Ancker, M. E., Bouwman, J., Wesselius, P. R., et al. 2000, A\&A, 357, 325

van den Ancker, M. E., Wesselius, P. R., \& Tielens, A. G. G. M. 2000, A\&A, 358, 1035

van Dishoeck, E. F., \& Blake, G. A. 1998, ARA\&A, 36, 317

Warren, S. G. 1984, Appl. Opt., 23, 1206

Waelkens, C., Malfait, K., \& Waters, L. B. F. M. 1997, Ap\&SS, 255,25

Waelkens, C., Waters, L. B. F. M., de Graaws, M. S., et al. 1996, A\&A, 315, 245

Willacy, K., \& Langer, W. D. 2000, ApJ, 544, 903

Wolfire, M., Tielens, A. G. G. M., \& Hollenbach, D. J. 1990, ApJ, 358, 116 